\title{
Equilibrium Selection in Global Games with Strategic Complementarities
}

\author{
David Frankel \\ Stephen Morris \\ Ady Pauzner \\ Tel Aviv University \\ Yale University \\ Tel Aviv University \\ January 26, 2000
}

\begin{abstract}
We study games with strategic complementarities, arbitrary numbers of players and actions, and slightly noisy payoff signals. We prove limit uniqueness: as the signal noise vanishes, the incomplete information game has a unique strategy profile that survives iterative dominance. This generalizes a result of Carlsson and van Damme for two player, two action games. The surviving profile, however, may depend on fine details of the structure of the noise. We provide sufficient conditions on payoffs for there to be noise-independent selection.
\end{abstract}

\section{Introduction}

In two player, two action games with common knowledge of payoffs, there often exist two strict Nash equilibria. Carlsson and van Damme [1993a] showed a remarkable result: if each player instead observes a noisy signal of the true payoffs, and if the $e x$ ante feasible payoffs include payoffs that make each action strictly dominant, then as the noise becomes small, iterative strict dominance eliminates all equilibria but one. 
In particular, if there are two Nash equilibria in the underlying complete information game, then the risk dominant equilibrium (Harsanyi and Selten [1988]) must be played in the game with noise.

Carlsson and van Damme's result can be reconstructed in two, logically separate, parts. First, there is a limit uniqueness result: as the noise in the incomplete information game becomes arbitrarily small, for almost any payoffs there is a unique action that survives iterative elimination of dominated strategies. The second is a noise independent selection result: as the noise goes to zero, the equilibrium played (for a given realization of the payoffs) is independent of the distribution of the noise.

In this paper, we show that Carlsson and van Damme's first result generalizes to many player, many action games with strategic complementarities. The second result, however, does not generalize: we present a counterexample (a two player, four action symmetric game) in which the equilibrium selected in the limit as the noise goes to zero does depend on the structure of the noise. However, we identify sufficient conditions under which noise independent selection does hold.

We consider the following setting. An unknown state of the world $\theta \in \mathbf{R}$ is drawn according to some prior. Each player $i$ observes a signal that is equal to $\theta+\nu \eta_{i}$, where $\nu>0$ is a scale factor and $\eta_{i}$ is a random variable with density $f_{i}$. Payoffs depend on players' actions and on $\theta$. Our substantive assumptions are (1) strategic complementarities: for any state $\theta$, each player's best response is increasing in the actions of her opponents; (2) single crossing: for any given opposing action profile, a player's best response is increasing in the state $\theta$; and (3) limit dominant actions: at sufficiently low (high) states $\theta$, each player's lowest (highest) action is strictly dominant. Call this incomplete information game $G(\nu)$. Under these and some technical continuity assumptions, we show that limit uniqueness holds: as the noise scale factor $\nu$ goes to zero, there is an essentially unique strategy profile surviving iterated deletion of dominated strategies (Theorem 1).

Without more stringent assumptions, however, there may not be noise-independent 
selection: the limiting equilibrium played at a state $\theta$ may depend on the noise densities $f_{i}$. We show that every local potential game with payoffs that are own-action concave has noise independent selection, i.e., a unique Nash equilibrium that is played in the limit of every noisy incomplete information game as the noise goes to zero. Local potential games include both the potential games of Monderer and Shapley [1996] and games with low p-dominant equilibria of Morris, Rob and Shin [1995] and Kajii and Morris [1997a]. In particular, local potential games include (1) all two player, two action games; (2) all many player, two action games with symmetric payoffs; and (3) all two player, three action games with symmetric payoffs. In each of these cases, we characterize the selected equilibrium.

\section{The Game}

The general game we analyze is as follows. There are $I \geq 2$ players, $1, \ldots, I$. A state $\theta \in \mathbf{R}$ is drawn from the real line according to a continuous and positive density $\phi$. Player $i$ observes a signal $x_{i}=\theta+\nu \eta_{i}$, where $\nu>0$ and each $\eta_{i}$ is distributed according to smooth density $f_{i}$ with support contained in the interval $\left[-\frac{1}{2}, \frac{1}{2}\right]$. Player $i$ 's action set is $A_{i}$, where $\{0,1\} \subseteq A_{i} \subseteq[0,1]$. We assume that either (a) each $A_{i}$ is discrete or (b) each $A_{i}$ is the continuum $[0,1] . \quad u_{i}(a, \theta)$ is player $i$ 's payoff if action profile $a$ is chosen and the state is $\theta$. Call this game $G(\nu)$.

Let $\Delta u_{i}\left(a_{i}, a_{i}^{\prime}, a_{-i}, \theta\right)$ be the difference in player $i$ 's utility from playing $a_{i}$ versus $a_{i}^{\prime}$ against $a_{-i}$ when the payoff parameter is $\theta: \Delta u_{i}\left(a_{i}, a_{i}^{\prime}, a_{-i}, \theta\right)=u_{i}\left(a_{i}, a_{-i}, \theta\right)-$ $u_{i}\left(a_{i}^{\prime}, a_{-i}, \theta\right)$. We make the following assumptions on the payoff functions:

A1 (Strategic Complementarities):

If $a_{i}>a_{i}^{\prime}$ and $a_{-i}>a_{-i}^{\prime}$, then (for all $\left.\theta\right) \Delta u_{i}\left(a_{i}, a_{i}^{\prime}, a_{-i}, \theta\right)>\Delta u_{i}\left(a_{i}, a_{i}^{\prime}, a_{-i}^{\prime}, \theta\right)$.

A2 (Limit Dominant Actions):

There exist constants $\underline{\theta}<\bar{\theta}$ such that $\Delta u_{i}\left(0, a_{i}, a_{-i}, \theta\right)>0$ if $a_{i} \neq 0$ and $\theta \leq \underline{\theta}$, and $\Delta u_{i}\left(1, a_{i}, a_{-i}, \theta\right)>0$ if $a_{i} \neq 1$ and $\theta \geq \bar{\theta}$. 
If each player's action space is finite, we can replace $\mathbf{A} 2$ by the weaker assumption:

A2' (Limit Unique Equilibrium):

There exist constants $\underline{\theta}<\bar{\theta}$ such that for all $\theta \notin[\underline{\theta}, \bar{\theta}]$, the complete information game in which each player $i$ 's payoffs are given by $u_{i}(\cdot, \theta)$ has a unique Nash equilibrium.

\section{A3 (Single Crossing):}

There is a $K_{0}>0$ such that for all $a_{i} \geq a_{i}^{\prime}$ and $\theta, \theta^{\prime} \in[\underline{\theta}, \bar{\theta}], \theta \geq \theta^{\prime}, \Delta u_{i}\left(a_{i}, a_{i}^{\prime}, a_{-i}, \theta\right)-$ $\Delta u_{i}\left(a_{i}, a_{i}^{\prime}, a_{-i}, \theta^{\prime}\right) \geq K_{0}\left(a_{i}-a_{i}^{\prime}\right)\left(\theta-\theta^{\prime}\right)$. (If the action space is continuous and $u_{i}$ is differentiable, a sufficient condition for $\mathbf{A} 3$ is that $\frac{\partial^{2} u_{i}\left(\left(a_{i}, a_{-i}\right), \theta\right)}{\partial a_{i} \partial \theta}>0$ for all $\theta \in[\underline{\theta}, \bar{\theta}]$.)

A4 (Continuity with Respect to $\theta$ ):

Each $u_{i}(a, \theta)$ is continuous with respect to $\theta$.

A5 (Continuity with Respect to Actions):

Each $u_{i}(a, \theta)$ is continuous with respect to $a$.

\section{A6 (Lipschitz):}

For all $\theta$ and $a_{-i}$, there exists a constant $K_{1}$ such that for all $a_{i}, a_{i}^{\prime}$,

$$
\left|\Delta u_{i}\left(a_{i}, a_{i}^{\prime}, a_{-i}, \theta\right)\right| \leq K_{1}\left(a_{i}-a_{i}^{\prime}\right)
$$

Also, for all $\theta$ there exists a constant $K_{2}$ such that for all $a_{i}, a_{i}^{\prime}, a_{-i}$, and $a_{-i}^{\prime}$,

$$
\left|\Delta u_{i}\left(a_{i}, a_{i}^{\prime}, a_{-i}, \theta\right)-\Delta u_{i}\left(a_{i}, a_{i}^{\prime}, a_{-i}^{\prime}, \theta\right)\right| \leq K_{2}\left(a_{i}-a_{i}^{\prime}\right) \sum_{j \neq i}\left(a_{j}-a_{j}^{\prime}\right)
$$

A strategy for player $i$ is a function $s_{i}: \mathbf{R} \rightarrow A_{i}$. A strategy profile is a vector of strategies, $s \equiv\left(s_{1}, \ldots, s_{I}\right)$. Strategy profile $s \equiv\left(s_{1}, \ldots, s_{I}\right)$ is increasing if $s_{i}\left(x_{i}\right)$ is weakly increasing in $x_{i}$ for all $i$; strategy profile $s$ is left continuous if $\lim _{x_{i}^{\prime} \uparrow x_{i}} s_{i}\left(x_{i}^{\prime}\right)=$ $s_{i}\left(x_{i}\right)$ for all $i$. Strategy profile $s^{\prime}$ is higher than strategy profile $s\left(s^{\prime} \geq s\right)$ if $s_{i}^{\prime}\left(x_{i}\right) \geq$ $s_{i}\left(x_{i}\right)$ for all $i=1, . ., I$ and $x_{i} \in \mathbf{R}$.

Finally, let $\tilde{\pi}_{i}\left(z \mid x_{i}, \nu\right)$ be player $i$ 's conditional density over the vector $z=\left(z_{j}\right)_{j \neq i}=$ $\left(\frac{x_{j}-x_{i}}{\nu}\right)_{j \neq i}$ of normalized differences in the signal errors when player $i$ observes signal $x_{i}$. 


\section{Uniqueness}

In this section we show that as the signal errors shrink to zero, iterative elimination of strictly dominated strategies selects an essentially unique Bayesian equilibrium of the game. We start with an intuition for the argument; a formal, general treatment follows.

\section{$3.1 \quad$ Intuition}

To see the intuition for limit uniqueness, consider the case of a symmetric two player game with a continuum of actions. Assume the two players have the same distribution of signal errors. Further assume that a player's payoff function is concave in her own action, so that her best response does not jump in response to small changes in her posterior distribution over the state $\theta$ and her opponent's action. Recall that a player's (pure) strategy is a function from her signal $x_{i}$ to an action $a_{i} \in[0,1]$. By the assumption of limit dominant actions (A2), we know that a player who observes a signal above some threshold must choose $a_{i}=1$. This means that no player will ever choose (i.e., put positive weight on) a pure strategy that lies below the following curve:

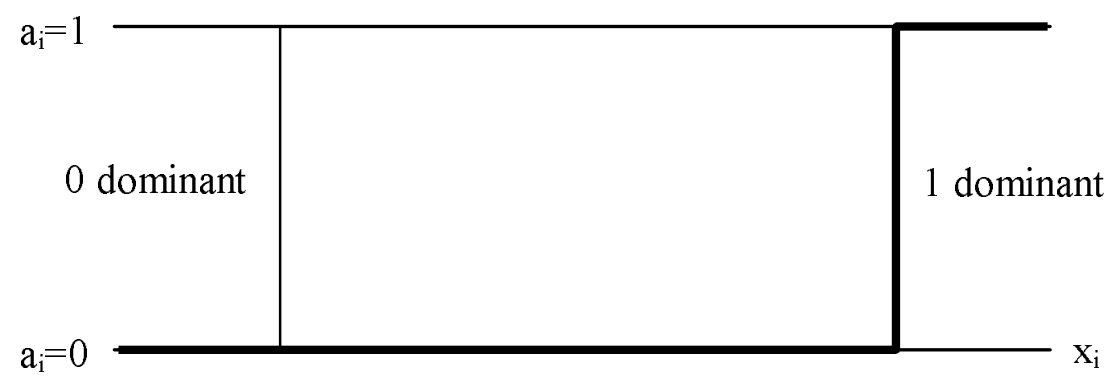

Knowing this, and because of the strategic complementarities, a player will never choose a strategy below the best response to this curve. So the best response to this curve gives a new lower bound on the strategies that can be selected. We iterate 
this process ad infinitum, and denote the limit by $S$. Note that $S$ is a symmetric equilibrium of the game.

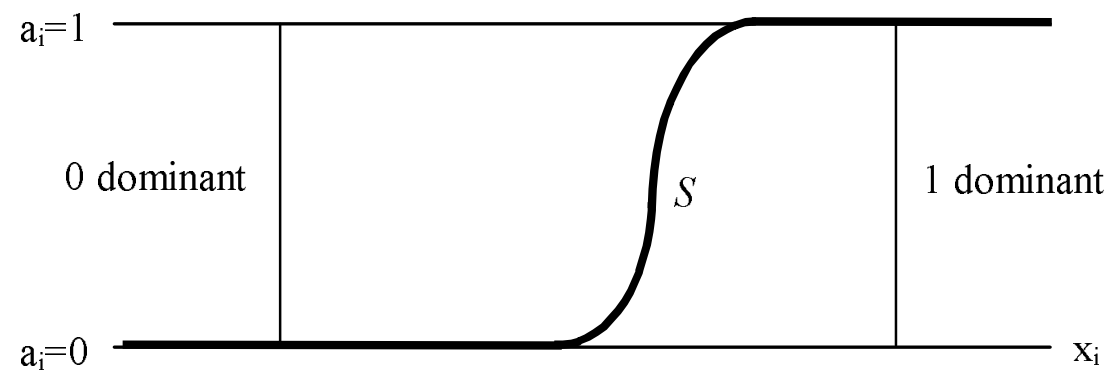

Since 0 is dominant for low enough signals, there is a translation $S_{0}$ of $S$ far enough to the left that a player will never choose a strategy that lies anywhere above $S_{0}$ :

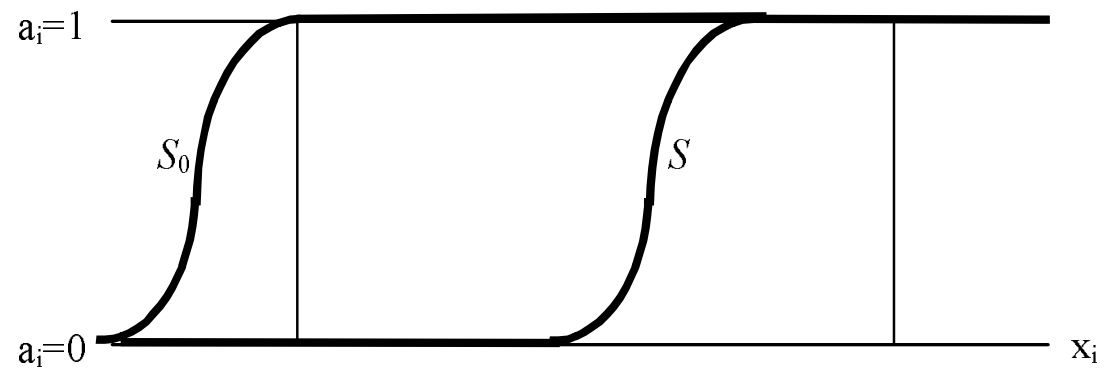

Now we do iterations starting from $S_{\mathbf{0}}$, but always using translations of $S_{\mathbf{0}}$. That is, we first find the rightmost translation $S_{1}$ of $S_{0}$ such that a player never strictly prefers to choose above $S_{1}$ if she thinks her opponent's strategy nowhere lies above $S_{0}$. Note that $S_{1}$ is the rightmost translation of $S_{0}$ that nowhere lies below the best response $\operatorname{BR}\left(S_{0}\right)$ to $S_{0}$ :

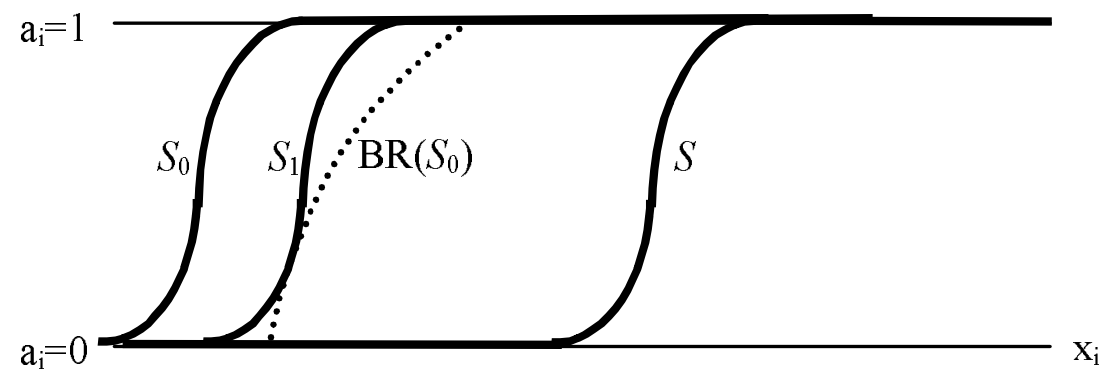


We iterate this procedure and denote the limit $S_{\infty}$. Clearly, any strategy ever chosen by any player must lie between $S_{\infty}$ and $S$.

Note that one more iteration from $S_{\infty}$ yields $S_{\infty}$ itself. This implies that the best response $\operatorname{BR}\left(S_{\infty}\right)$ lies entirely to the right of $S_{\infty}$ and touches $S_{\infty}$ at at least one point. Let $P$ denote this point, and let $x$ be the signal that corresponds to $P$. Let $P^{\prime}$ be the point on $S$ that is at the same height as $P$; let $x^{\prime}$ be the signal that corresponds to $P^{\prime}$

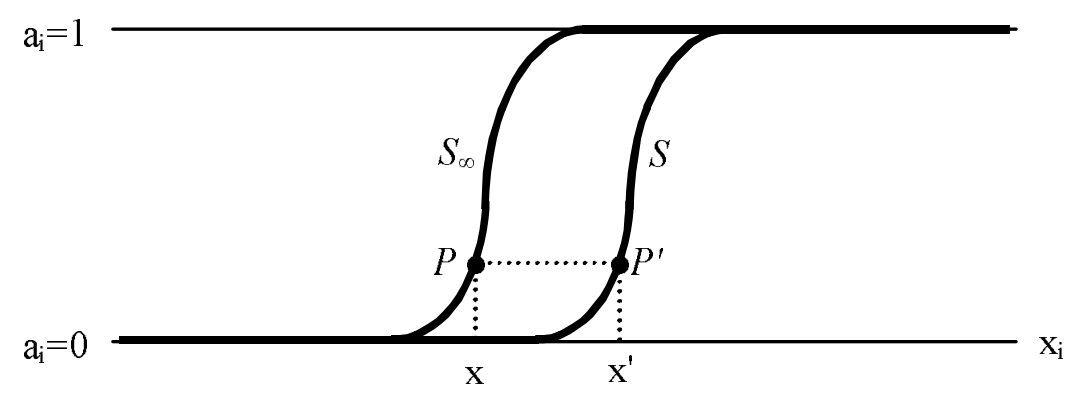

We now exploit the fact that the signal errors are small. This means that a player $i$ can be sure that the payoff parameter $\theta$ is very close to her signal $x_{i}$. Consequently, the prior over $\theta$ is approximately uniform for values of $\theta$ that are possible given her signal. (Recall that we assume a smooth prior over $\theta$.) Thus, the posterior of a player with signal $x$ over the error in her signal, $x-\theta$, is approximately the same as the posterior of a player with signal $x^{\prime}$ over the error in her signal, $x^{\prime}-\theta$. Since the signal error of the player's opponent is independent of $\theta$, the player's posterior over the difference between her signal error and that of her opponent is also about the same if her signal is $x$ as if her signal is $x^{\prime}$. But the difference between the two players signal errors is just the difference between their signals: $\left(x_{i}-\theta\right)-\left(x_{j}-\theta\right)=x_{i}-x_{j}$. Thus, a player's posterior over the difference between her signal and that of her opponent is about the same at $x$ as at $x^{\prime}$. Thus, since $S_{\infty}$ is an exact translation of $S$, a player who observes $x$ and thinks that her opponent will play according to $S_{\infty}$ expects about the same action distribution as a player who observes $x^{\prime}$ and thinks that the opponent will play according to $S$. By construction, both players to want to play the same 
action: $P$ and $P^{\prime}$ are at the same height. Since a player's optimal action is strictly increasing in her estimate of $\theta$ (controlling for her opponent's action distribution), $x$ and $x^{\prime}$ must coincide - otherwise, the player who observes $x^{\prime}$ would want to play a higher action than a player who observes $x$. But this means that the curves $S_{\infty}$ and $S$ must also coincide: a unique equilibrium survives iterative strict dominance.

\subsection{Formal Result}

We now turn to the formal treatment of many player, many action games.

Theorem $1 G(\nu)$ has an essentially unique increasing strategy profile surviving iterative strict dominance in the limit as $\nu \rightarrow 0$, i.e., there exists strategy profile $s^{*}$ such if $s^{\nu}$ is a sequence of equilibria satisfying iterative strict dominance in $G(\nu)$, then $s_{i}^{\nu}\left(x_{i}\right) \rightarrow s_{i}^{*}\left(x_{i}\right)$ for almost all $x_{i} \in \mathbf{R}$.

Proof. The proof has two parts. In the first (Lemma 2), we consider a simplified game in which an agent's prior over $\theta$ is uniform and her payoff depends directly on her signal rather than on $\theta$. Because of the uniform prior, a player's posterior over the difference between her signal and those of other players is independent of her signal, so we obtain uniqueness even when the noise is large. The argument generalizes the "iterate with translations" approached used in the above intuition.

In the second part of the proof, we show that the original game "converges" to the simplified one as the signal errors shrink. That is, a player's posterior over the differences between her signal and those of other players becomes approximately independent of her own signal (Lemma 3). Moreover, in the limit it does not matter whether a player's payoffs depend on her signal or on $\theta$ since these become arbitrarily close. As a result, the strategy profiles surviving iterative dominance in the original and simplified games converge to each other (Lemma 4).

The simplified game is defined as follows. Let the state $\theta$ be drawn uniformly from some large interval that includes $[\underline{\theta}, \bar{\theta}]$ and let player $i$ 's payoff depend on her signal 
$x_{i}$ instead of the state. Thus $u_{i}\left(a, x_{i}\right)$ is player $i$ 's payoff if action profile $a$ is chosen and she observes signal $x_{i}$. Call this game $G^{*}(\nu)$. Note that with a uniform prior on states, player $i$ 's posterior $\pi_{i}(z)$ over the vector $z=\left(z_{j}\right)_{j \neq i}=\left(\frac{x_{j}-x_{i}}{\nu}\right)_{j \neq i}$ of normalized differences between other players' signals and her own signal is independent of $x_{i}$ and $\nu$.

Lemma 2 shows that each game $G^{*}(\nu)$ has an essentially unique strategy profile surviving iterative strict dominance, which is weakly increasing.

Lemma 2 There exists a weakly increasing strategy profile $s^{*}$ such that any profile $s$ that survives iterative strict dominance must (a) be weakly increasing in $x_{i}$ for all $i$ and (b) agree with $s_{i}^{*}$ except perhaps at the (at most countably many) signals $x_{i}$ at which $s_{i}^{*}\left(x_{i}\right)$ is discontinuous.

Proof. See appendix.

Lemma 3 shows that as $\nu \rightarrow 0$, players' posteriors over differences in the signal errors in $G(\nu), \tilde{\pi}_{i}\left(z \mid x_{i}, \nu\right)$, converge to the posteriors that would result from a uniform prior distribution over $\theta$. For any probability measure $\mu$ on $\mathbf{R}^{I-1}$, let $\Psi_{\varepsilon}(\mu)$ be the set of probability measures that differ from $\mu$ by no more than $\varepsilon$ for any event:

$$
\Psi_{\varepsilon}(\mu) \equiv\left\{\mu^{\prime}: \sup _{E \subseteq \mathbf{R}^{I-1}}\left|\mu(E)-\mu^{\prime}(E)\right| \leq \varepsilon\right\} .
$$

Lemma 3 For any $\varepsilon>0$ and compact interval $B$, there exists $\bar{\nu}>0$ such that $\tilde{\pi}_{i}\left(\cdot \mid x_{i}, \nu\right) \in \Psi_{\varepsilon}\left(\pi_{i}(\cdot)\right)$ for all $x_{i} \in B$ and all $\nu \leq \bar{\nu}$.

Proof. See appendix.

Lemma 4 uses the above results to show that as the signal noise shrinks, agents' behavior in $G(\nu)$ converges to the unique outcome of $G^{*}(\nu)$. Write $\underline{s}^{\nu}\left(\bar{s}^{\nu}\right)$ for the left (right) continuous version of the essentially unique equilibrium of the game $G^{*}(\nu)$. 
Lemma 4 For any $\varepsilon>0$, there exists $\bar{\nu}>0$ such that for all $\nu \leq \bar{\nu}$ and any strategy profile s of $G(\nu)$ surviving iterated deletion of strictly dominated strategies,

$$
\bar{s}_{i}^{\nu}\left(x_{i}+\varepsilon\right) \geq s_{i}\left(x_{i}\right) \geq \underline{s}_{i}^{\nu}\left(x_{i}-\varepsilon\right)
$$

Proof. See appendix.

Theorem 1 follows immediately from Lemmas 2 and 4 . Q.E.D.

\section{Noise Independent Selection: A Counterexam- ple}

In showing limit uniqueness, we began with a given noise structure and scaled it down by taking the scale factor $\nu$ to zero. Our result does not imply that the selected equilibrium is independent of the structure of the noise (i.e., of the densities $\left.f_{i}\right)$.

To obtain some intuition for when the selected equilibrium will be independent of the structure of the noise, suppose the game has two players, each with the same discrete action set $\bar{A}$ and the same payoff function, and each player's noise term has the same symmetric distribution $f$. Assume that each player follows the same increasing strategy, $s: \mathbf{R} \rightarrow \bar{A}$. As $\nu \rightarrow 0$, what beliefs does each player have over the action of her opponent at the critical point where she switches from one action to another? Recall that for small $\nu$, each player's posterior belief about the other's signal is computed approximately as if she had a uniform prior over $\theta$.

Suppose first that there are two actions, so that

$$
s(x)=\left\{\begin{array}{l}
0, \text { if } x<c \\
1, \text { if } x \geq c
\end{array}\right.
$$

A player observing signal $c$ will assign probability $\frac{1}{2}$ to her opponent's choosing action 0 and probability $\frac{1}{2}$ to her opponent's choosing action 1 . This is independent of the 
choice of $c$ and the distribution $f$. Thus as the noise goes to zero, $c$ must converge to the payoff parameter at which the player is indifferent between the two actions if she has a 50/50 conjecture over her opponent's action. This is simply the symmetric version of Carlsson and van Damme's (1993) result.

Now suppose that there are three actions, $0,1 / 2$, and 1 , so that

$$
s(x)=\left\{\begin{array}{l}
0, \text { if } x<c_{1} \\
\frac{1}{2}, \text { if } c_{1} \leq x<c_{2} \\
1, \text { if } c_{2} \leq x
\end{array}\right.
$$

A player observing signal $c_{1}$ will assign probability $\frac{1}{2}$ to her opponent choosing action 0 , some probability $\lambda$ to her opponent choosing action $\frac{1}{2}$, and probability $\frac{1}{2}-\lambda$ to her opponent choosing action 1 ; a player observing signal $c_{2}$ will assign probability $\frac{1}{2}-\lambda$ to her opponent choosing action 0 , probability $\lambda$ to her opponent choosing action $\frac{1}{2}$ and probability $\frac{1}{2}$ to her opponent choosing action 1 . For any distribution $f$, we can choose $c_{1}$ and $c_{2}$ so that $\lambda$ takes any value in $[0,1 / 2]$. In other words, the distribution of noise does not effect the limiting conjectures that each player may end up having over her opponent's actions.

This implies noise independent selection: any profile $\left(c_{1}, c_{2}\right)$ that is an equilibrium as $\nu \rightarrow 0$ under a noise structure $f$ must also be an equilibrium under any other noise structure $f^{\prime}$. To see why, let us distinguish between two cases. In the first, $c_{1}-c_{2}$ does not shrink to 0 as $\nu \rightarrow 0$. This means that $\lambda$ converges to 1: for sufficiently small $\nu$, a player with signal $c_{1}$ is indifferent between 0 and $1 / 2$ and thinks that her opponent will play 0 or $1 / 2$ with equal probabilities; a player with signal $c_{2}$ is indifferent between $1 / 2$ and 1 and puts equal probabilities on her opponent's playing $1 / 2$ and 1 . These signals clearly must converge to particular payoff parameters, independent of the structure of the signal errors (since the player's beliefs are independent of $f$ ).

In the second case, $\lim _{\nu \rightarrow 0}\left(c_{1}-c_{2}\right)=0$. Here, $\lambda$ need not converge to 1 . But if we replace the signal error structure $f$ with some other structure $f^{\prime}$, we can construct an equilibrium near the one given by $f$ by simply adjusting the gap between $c_{1}$ and 
$c_{2}$ so as to keep $\lambda$ the same under $f^{\prime}$ as under $f$. Since the gap between $c_{1}$ and $c_{2}$ asymptotically shrinks to zero, we can make this adjustment without changing the limit to which both cutoffs converge. Thus, under $f^{\prime}$ there is a sequence of equilibria of the incomplete information game that converges to the same limit as the sequence of equilibria under $f$. This explains why there is noise-independent selection. In the next section, we show that there is selection is noise-independent for two player, three action, symmetric-payoff games, even with general asymmetric noise distributions $f_{i}$.

With four or more actions, the above property ceases to hold. The set of conjectures a player can have over her opponent's action does depend on the structure of the noise. We now present a counterexample with four actions, in which the equilibrium selected in the limit depends on the noise. Let $I=2, A_{1}=A_{2}=\left\{0, \frac{1}{3}, \frac{2}{3}, 1\right\}$ and let $G(\nu)$ and $\widehat{G}(\nu)$ be two games satisfying the assumptions of Section 2; these two games are identical except that in $G(\nu), \eta_{1}$ and $\eta_{2}$ are distributed according to the density

$$
f(\eta)=1
$$

on the interval $\left[-\frac{1}{2}, \frac{1}{2}\right]$; in $\widehat{G}(\nu), \eta_{1}$ and $\eta_{2}$ are distributed according to the density

$$
\widehat{f}(\eta)=2-4|\eta|
$$

on the interval $\left[-\frac{1}{2}, \frac{1}{2}\right]$. Note that under a uniform prior on $\theta$, the resulting symmetric distributions of $z=\eta_{1}-\eta_{2}$ have support $[-1,1]$ and densities

$$
\begin{aligned}
& \pi(z)=1-|z| \\
& \widehat{\pi}(z)=\left\{\begin{array}{l}
2(1+z)^{2}, \text { if }-1 \leq z \leq-\frac{1}{2} \\
1-2 z^{2}, \text { if }-\frac{1}{2} \leq z \leq \frac{1}{2} \\
2(1-z)^{2}, \text { if } \frac{1}{2} \leq z \leq 1
\end{array}\right.
\end{aligned}
$$


Assume that $u\left(\cdot, \theta^{*}\right)=g^{*}(\cdot)$, where $g^{*}$ is given by the following symmetric matrix:

\begin{tabular}{|l|l|l|l|l|}
\hline$g^{*}$ & 0 & $\frac{1}{3}$ & $\frac{2}{3}$ & 1 \\
\hline 0 & 2000,2000 & 1936,1656 & 1144,1056 & 391,254 \\
\hline$\frac{1}{3}$ & 1656,1936 & 2000,2000 & 1600,1800 & 1245,1000 \\
\hline$\frac{2}{3}$ & 1056,1144 & 1800,1600 & 2000,2000 & 1660,2160 \\
\hline 1 & 254,391 & 1000,1245 & 2160,1660 & 2000,2000 \\
\hline
\end{tabular}

One may verify that this is a game of strategic complementarities, since each row of the following table is increasing.

\begin{tabular}{|l|l|l|l|l|}
\hline$g_{1}^{*}\left(a_{1}+\frac{1}{3}, a_{2}\right)-g_{1}^{*}\left(a_{1}, a_{2}\right)$ & $a_{2}=0$ & $a_{2}=\frac{1}{3}$ & $a_{2}=\frac{2}{3}$ & $a_{2}=1$ \\
\hline$a_{1}=0$ & -344 & 64 & 456 & 854 \\
\hline$a_{1}=\frac{1}{3}$ & -600 & -200 & 400 & 415 \\
\hline$a_{1}=\frac{2}{3}$ & -802 & -800 & 160 & 340 \\
\hline
\end{tabular}

(Also note that payoffs are concave in each player's own action, since each column in the above table is decreasing.) Let strategy profile $\hat{s}[k]$ be defined by:

$$
\widehat{s}_{i}[k]\left(x_{i}\right)=\left\{\begin{array}{l}
0, \text { if } x_{i}<k-\frac{1}{4} \\
\frac{1}{3}, \text { if } k-\frac{1}{4} \leq x_{i}<k \\
\frac{2}{3}, \text { if } k \leq x_{i}<k+\frac{1}{4} \\
1, \text { if } k+\frac{1}{4} \leq x_{i}
\end{array}\right.
$$

Lemma 5 Let the interaction structure be given by $\widehat{\pi}$. There exists $\widehat{\varepsilon}>0$ and $\widehat{\delta}>0$ such that if $\theta \in\left[\theta^{*}-\widehat{\delta}, \theta^{*}+\hat{\delta}\right]$ and payoffs are always given by $u\left(\cdot, \theta^{*}\right)$, the best response to strategy profile $\hat{s}[k]$ is less than or equal to $\widehat{s}[k+\widehat{\varepsilon}]$.

PROOF. If player 1 observes $x_{1}$, she believes that $x_{2}-x_{1}$ is distributed according to $\hat{\pi}$. If she believes that her opponent is following strategy $\hat{s}_{2}[k]$, her conjectures 
over her opponents' actions are the following:

\begin{tabular}{|l|l|l|l|l|l|}
\cline { 2 - 6 } Player 1's Signal & & $a_{2}=0$ & $a_{2}=\frac{1}{3}$ & $a_{2}=\frac{2}{3}$ & $a_{2}=1$ \\
\cline { 2 - 6 } & $k-\frac{1}{4}$ & $\frac{1}{2}$ & $\frac{23}{96}$ & $\frac{17}{96}$ & $\frac{1}{12}$ \\
\cline { 2 - 6 } & $k$ & $\frac{25}{96}$ & $\frac{23}{96}$ & $\frac{23}{96}$ & $\frac{25}{96}$ \\
\cline { 2 - 6 } & $k+\frac{1}{4}$ & $\frac{1}{12}$ & $\frac{17}{96}$ & $\frac{23}{96}$ & $\frac{1}{2}$ \\
\hline
\end{tabular}

One may verify that if player 1 observes signal $k-\frac{1}{4}$ and has payoffs given by $u\left(\cdot, \theta^{*}\right)$, then she strictly prefers action 0 to action $\frac{1}{3}\left(\right.$ since $\frac{1}{2}(-344)+\frac{23}{96}(64)+\frac{17}{96}(456)+$ $\left.\frac{1}{12}(854)=-\frac{19}{4}<0\right)$. Similarly, if player 1 observes signal $k$ and has payoffs given by $u\left(\cdot, \theta^{*}\right)$, she strictly prefers action $\frac{1}{3}$ to action $\frac{2}{3}$ (since $\frac{25}{96}(-600)+\frac{23}{96}(-200)+$ $\left.\frac{23}{96}(400)+\frac{25}{96}(415)=-\frac{25}{96}<0\right)$ and if player 1 observes signal $k+\frac{1}{4}$ and has payoffs given by $u\left(\cdot, \theta^{*}\right)$, she strictly prefers action $\frac{2}{3}$ to action 1 . By continuity, these strict preferences will be maintained for signals in a small neighborhoods of those cutoff points and for payoffs in a small neighborhood of $u\left(\cdot, \theta^{*}\right)$.

Now consider the game $\widehat{G}^{D}\left(\nu, \theta^{*}\right)$, which is like $\widehat{G}^{*}(\nu)$, except that the payoff functions are replaced by:

$$
u_{i}^{D}(a, \theta)=\left\{\begin{array}{l}
u_{i}(a, \underline{\theta}), \text { if } \theta \leq \underline{\theta} \\
u_{i}\left(a, \theta^{*}+\widehat{\delta}\right), \text { if } \underline{\theta} \leq \theta \leq \theta^{*}+\widehat{\delta} \\
u_{i}(a, \bar{\theta}), \text { if } \theta \geq \theta^{*}+\widehat{\delta}
\end{array}\right.
$$

Corollary 6 In the game $\widehat{G}^{D}\left(\nu, \theta^{*}\right)$, any strategy s satisfying iterated deletion of strictly dominated strategies satisfies $s \leq \widehat{s}\left[\theta^{*}+\widehat{\delta}-\nu\right]$; thus $s_{i}\left(x_{i}\right)=0$ for all $x_{i} \leq$ $\theta^{*}+\widehat{\delta}-2 \nu$

PROOF. By induction, verify that if strategy profile $s$ survives $k+1$ rounds of deletion of strictly dominated strategies, then $s \leq \widehat{s}\left[\max \left(\underline{\theta}-\nu+k \widehat{\varepsilon}, \theta^{*}+\widehat{\delta}-\nu\right)\right]$.

Now we have:

Lemma 7 If $s^{\nu}$ is the essentially unique equilibrium of $\widehat{G}^{*}(\nu)$, then $s_{i}^{\nu}\left(x_{i}\right)=0$ for all $x_{i} \leq \theta^{*}+\widehat{\delta}-\nu$. 
But now let strategy profile $s[k]$ be defined by:

$$
s_{i}[k]\left(x_{i}\right)=\left\{\begin{array}{l}
0, \text { if } x_{i}<k-\frac{7}{25} \\
\frac{1}{3}, \text { if } k-\frac{7}{25} \leq x_{i}<k \\
\frac{2}{3}, \text { if } k \leq x_{i}<k+\frac{7}{25} \\
1, \text { if } \frac{7}{25} \leq k+x_{i}
\end{array}\right.
$$

Lemma 8 Let the interaction structure be given by $\pi$. There exists $\varepsilon>0$ and $\delta>0$ such that if $\theta \in\left[\theta^{*}-\delta, \theta^{*}+\delta\right]$ and payoffs were always given by $u\left(\cdot, \theta^{*}\right)$, the best response to strategy profile $s[k]$ is more than or equal to $s[k-\varepsilon]$.

PROOF. If player 1 observes $x_{1}$, she believes that $x_{2}-x_{1}$ is distributed according to $\pi$. If she believes that her opponent is following strategy $\widehat{s}_{2}[k]$, her conjectures over her opponents' actions are the following:

\begin{tabular}{|l|l|l|l|l|l|}
\hline & $a_{2}=0$ & $a_{2}=\frac{1}{3}$ & $a_{2}=\frac{2}{3}$ & $a_{2}=1$ \\
\cline { 2 - 6 } Player 1's Signal & $k-\frac{7}{25}$ & $\frac{1}{2}$ & $\frac{301}{1250}$ & $\frac{203}{1250}$ & $\frac{121}{1250}$ \\
\cline { 2 - 7 } & $k$ & $\frac{162}{625}$ & $\frac{301}{1250}$ & $\frac{301}{1250}$ & $\frac{162}{625}$ \\
\cline { 2 - 6 } & $k+\frac{7}{25}$ & $\frac{121}{1250}$ & $\frac{203}{1250}$ & $\frac{301}{1250}$ & $\frac{1}{2}$ \\
\hline
\end{tabular}

One may verify that if player 1 observes signal $k-\frac{7}{25}$ and her payoffs are given by $u\left(\cdot, \theta^{*}\right)$, then she strictly prefers action $\frac{1}{3}$ to action $0\left(\right.$ since $\frac{1}{2}(-344)+\frac{301}{1250}(64)+$ $\left.\frac{203}{1250}(456)+\frac{121}{1250}(854)=\frac{83}{625}>0\right)$. Similarly, if player 1 observes signal $k$ and her payoffs are given by $u\left(\cdot, \theta^{*}\right)$, she strictly prefers action $\frac{2}{3}$ to action $\frac{1}{3}$ and if player 1 observes signal $k+\frac{7}{25}$ and her payoffs are given by $u\left(\cdot, \theta^{*}\right)$, she strictly prefers action 1 to action $\frac{2}{3}$. By continuity, these strict preferences will be maintained for signals in a small neighborhoods of those cutoff points and for payoffs in a small neighborhood of $u\left(\cdot, \theta^{*}\right)$.

But now mimicking the above argument, we have:

Lemma 9 If $s^{\nu}$ is the essentially unique equilibrium of $G^{*}(\nu)$, then $s_{i}^{\nu}\left(x_{i}\right)=1$ for all $x_{i} \geq \theta^{*}-\delta+\nu$. 


\section{A Sufficient Condition for Noise-Independent Se- lection}

In this section we show that there is noise-independent selection at some parameter $\theta$ if (a) payoffs at $\theta$ are own-action concave and (b) the complete information game with these payoffs has a strategy profile that is a local potential maximizer. This strategy profile must be played in the limit, regardless of the structure of signal errors.

Let $H$ be the set of complete information games $\mathbf{g}=\left(g_{1}, \ldots, g_{I}\right), g_{i}: A \rightarrow \mathbf{R}$. Let $F$ be the set of noise structures $\mathbf{f}=\left(f_{1}, \ldots, f_{I}\right)$, where each $f_{i}$ is a smooth density on the interval $\left[-\frac{1}{2}, \frac{1}{2}\right]$. Our arguments so far have established limit uniqueness, which can be restated as follows:

Corollary 10 There exists an assignment $\widetilde{a}: H \times F \rightarrow A$ of action profiles to complete information games and noise structures such that for any incomplete information game $G(\nu)$ as defined above, if $s^{*}$ is the limit, as $\nu \rightarrow 0$, of any sequence of strategy profiles that survive iterative strict dominance in $G(\nu)$, then $s^{*}(x)=$ $\widetilde{a}\left(\left\{u_{i}\left(., x_{i}\right)\right\}_{i=1}^{I}, \mathbf{f}\right)$ for almost all $x$.

Thus except at jump points, the action profile that players must play in the limit at any state $\theta$ is uniquely determined by the payoffs at that state and the noise structure. There is no multiplicity of equilibria (unlike in the complete information case), but the equilibrium played may depend on the noise structure.

For some games, it is possible to identify the limiting behavior independently of the noise structure. In other words, for some games $\mathbf{g} \in H, \widetilde{a}(\mathbf{g}, \mathbf{f})=a^{*}$ for all $\mathbf{f} \in F$. In this case, we say that $a^{*}$ is the noise independent selection of $\mathbf{g}$. We will give a sufficient condition for this to hold.

If $A_{i}$ is a discrete action set, write $a_{i}^{+}$for the next action above $a_{i}$ and $a_{i}^{-}$for the next action below $a_{i}$. We now define own-action concavity and the notion of a local potential maximizer. 
Definition 11 The complete information game $g$ with discrete action sets is ownaction-concave if $g_{i}\left(a_{i}^{+}, a_{-i}\right)-g_{i}\left(a_{i}, a_{-i}\right) \leq g_{i}\left(a_{i}, a_{-i}\right)-g_{i}\left(a_{i}^{-}, a_{-i}\right)$ for all $a_{i} \in$ $A_{i} \backslash\left\{\underline{a}_{i}, \bar{a}_{i}\right\}$ and $a_{-i} \in A_{-i}$. The complete information game $g$ with continuous action sets $A_{i}=[0,1]$ is own-action concave if, for any $\varepsilon>0, g_{i}\left(a_{i}+\varepsilon, a_{-i}\right)-g_{i}\left(a_{i}, a_{-i}\right)$ is weakly decreasing in $a_{i} \in(0,1-\varepsilon)$.

Definition 12 Action profile $a^{*}$ is a local potential maximizer (LP-maximizer) of $g$ if there exists a local potential function $v: A \rightarrow \mathbf{R}$ with $v\left(a^{*}\right)>v(a)$ for all $a \neq a^{*}$ and, for each $i$, a function $\mu_{i}: A_{i} \rightarrow \mathbf{R}_{+}$, such that for all $i=1, . ., I$ and $a_{-i} \in A_{-i}$,

$$
\begin{aligned}
v\left(a_{i}, a_{-i}\right)-v\left(a_{i}^{-}, a_{-i}\right) & \geq \mu_{i}\left(a_{i}\right)\left[g_{i}\left(a_{i}, a_{-i}\right)-g_{i}\left(a_{i}^{-}, a_{-i}\right)\right] \text { if } a_{i}>a_{i}^{*} \\
\text { and } v\left(a_{i}, a_{-i}\right)-v\left(a_{i}^{+}, a_{-i}\right) & \geq \mu_{i}\left(a_{i}\right)\left[g_{i}\left(a_{i}, a_{-i}\right)-g_{i}\left(a_{i}^{+}, a_{-i}\right)\right] \text { if } a_{i}<a_{i}^{*}
\end{aligned}
$$

if $A_{i}$ is discrete. If $A_{i}$ is continuous, there must be a constant $c_{i}$, independent of $a_{i}$ and $a_{-i}$, such that if $\delta \in\left[0, c_{i}\right]$ then

$$
\begin{aligned}
v\left(a_{i}+\delta, a_{-i}\right)-v\left(a_{i}, a_{-i}\right) & \geq \mu_{i}\left(a_{i}\right)\left[g_{i}\left(a_{i}+\delta, a_{-i}\right)-g_{i}\left(a_{i}, a_{-i}\right)\right] \text { if } a_{i}>a_{i}^{*} \\
\text { and } v\left(a_{i}, a_{-i}\right)-v\left(a_{i}+\delta, a_{-i}\right) & \geq \mu_{i}\left(a_{i}\right)\left[g_{i}\left(a_{i}, a_{-i}\right)-g_{i}\left(a_{i}+\delta, a_{-i}\right)\right] \text { if } a_{i}<a_{i}^{*}
\end{aligned}
$$

Importantly, the LP-maximizer property guarantees that a profile is a strict Nash equilibrium if payoffs are own-action concave:

Lemma 13 If $a^{*}$ is an LP-maximizer and $\mathbf{g}$ is own-action concave, then $a^{*}$ is a strict Nash equilibrium.

Proof. Consider any player $i$ and any alternative action $a_{i}$. WLOG assume $a_{i}>a_{i}^{*}$. In the continuous case, for any $\delta \in\left[0, c_{i}\right]$,

$$
0>v\left(a_{i}^{*}+\delta, a_{-i}^{*}\right)-v\left(a_{i}^{*}, a_{-i}^{*}\right) \geq \mu_{i}\left(a_{i}^{*}\right)\left(g_{i}\left(a_{i}^{*}+\delta, a_{-i}^{*}\right)-g_{i}\left(a_{i}^{*}, a_{-i}^{*}\right)\right)
$$

so $g_{i}\left(a_{i}^{*}+\delta, a_{-i}^{*}\right)<g_{i}\left(a_{i}^{*}, a_{-i}^{*}\right)$. By own-action concavity, $g_{i}\left(a_{i}^{*}, a_{-i}^{*}\right)$ exceeds $g_{i}\left(a_{i}, a_{-i}^{*}\right)$ for any $a_{i}>a_{i}^{*}$. An analogous argument shows the same for any $a_{i}<a_{i}^{*}$. The proof is analogous for the case of discrete actions. 
Proposition 14 states that if, at some state $\theta$, payoffs are own-action concave and have an LP-maximizer, then that LP-maximizer must be played in the limit as the signal errors shrink, regardless of the noise structure.

Proposition 14 If $s^{*}$ is the essentially unique limiting equilibrium of $G(\nu)$ and $a^{*}$ is an LP-maximizer of the own-action concave complete information game $\left(g_{i}\left(\cdot, \theta^{*}\right)\right)_{i=1}^{I}$, then $s^{*}\left(\theta^{*}\right)=a^{*}$.

Proof. Let $\underline{g}_{i}$ be some particular payoff function for player $i$ with the property that action 0 is a dominant strategy. Fix some $\theta^{*} \in \mathbf{R}$ and consider the incomplete information game $G^{* *}\left(\nu, \theta^{*}\right)$ with uniform prior, noise structure $\mathbf{f}$ and payoff functions

$$
\underline{g}_{i}\left(a, x_{i}\right)=\left\{\begin{array}{l}
g_{i}\left(a, \theta^{*}\right), \text { if } x_{i} \geq \theta^{*} \\
\underline{g}_{i}(a), \text { if } x_{i}<\theta^{*}
\end{array}\right.
$$

Suppose that action profile $a^{*}=\left(a_{i}^{*}\right)_{i=1}^{I}$ is an LP-maximizer of the complete information game $g=\left(g_{i}\left(\cdot, \theta^{*}\right)\right)_{i=1}^{I}$ and that this game is own-action concave.

For now, fix $\nu=1$. We will be interested in left-continuous, weakly increasing strategies for player $i$ where she never chooses an action above $a_{i}^{*}$. Any such strategy can be represented by a function $\zeta_{i}:\left[0, a_{i}^{*}\right] \rightarrow \mathbf{R}$ where $\zeta_{i}\left(a_{i}\right)$ is the highest signal at which an action less than or equal to $a_{i}$ is played.

We wish to define the unique left-continuous best response to strategy profile $\zeta$ in the game $G^{* *}\left(\nu, \theta^{*}\right)$. Note that the assumpion that $a^{*}$ is an LP-maximizer (and thus, by lemma 13, a strict Nash equilibrium) and the strategic complementarities assumption imply that the best response to $\zeta$ will itself involve each player $i$ choosing an action less than or equal to $a_{i}^{*}$. We write $\beta(\zeta)=\left(\beta_{i}(\zeta)\right)_{i=1}^{I}$ for this best response.

To give an explicit expression for $\beta_{i}(\zeta)$, first write $s_{i}^{\zeta_{i}}$ for player $i$ 's strategy written in standard notation, i.e., $s_{i}^{\zeta_{i}}\left(x_{i}\right)=\min \left\{a_{i}: \zeta_{i}\left(a_{i}\right) \geq x_{i}\right\}$. (It is correct to take the min because of left continuity.) Write $s_{-i}^{\zeta_{-i}}\left(x_{-i}\right)=\left(s_{j}^{\zeta_{j}}\left(x_{j}\right)\right)_{j \neq i}$ and $s^{\zeta}(x)=\left(s_{j}^{\zeta_{j}}\left(x_{j}\right)\right)_{j=1}^{I}$. Now if player $i$ observes $x_{i}<\theta^{*}$, action 0 is dominant. 
If player $i$ observes $x_{i} \geq \theta^{*}$, her payoff to choosing action $a_{i}$, if she believes her opponents are following strategies $\zeta_{-i}$, is

$$
\int_{\theta \in \mathbf{R}} \int_{x_{-i} \in \mathbf{R}^{I-1}} g_{i}\left(\left(a_{i}, s_{-i}^{\zeta_{-i}}\left(x_{-i}\right)\right), x_{i}\right)\left(\prod_{j=1}^{I} f_{j}\left(x_{j}-\theta\right)\right) d x_{-i} d \theta .
$$

Thus an action less than or equal to $a_{i}$ is a best response if

$$
\min \left\{\underset{a_{i}^{\prime} \in A_{i}}{\arg \max } \int_{\theta \in \mathbf{R}} \int_{x_{-i} \in \mathbf{R}^{I-1}} g_{i}\left(\left(a_{i}^{\prime}, s_{-i}^{\zeta_{-i}}\left(x_{-i}\right)\right), x_{i}\right)\left(\prod_{j=1}^{I} f_{j}\left(x_{j}-\theta\right)\right) d x_{-i} d \theta\right\} \leq a_{i} .
$$

Recall that $\zeta_{i}\left(a_{i}\right)$ is the largest value of $x_{i}$ at which an action less than or equal to $a_{i}$ is played under $i$ 's strategy. Thus $\beta_{i}(\zeta)\left(a_{i}\right)$ is the maximum of $\theta^{*}$ and

$$
\max \left\{x_{i}: \min \left\{\underset{a_{i}^{\prime} \in A_{i}}{\arg \max } \int_{\theta \in \mathbf{R}} \int_{x_{-i} \in \mathbf{R}^{I-1}} g_{i}\left(\left(a_{i}^{\prime}, s_{-i}^{\zeta}\left(x_{-i}\right)\right), x_{i}\right)\left(\prod_{j=1}^{I} f_{j}\left(x_{j}-\theta\right)\right) d x_{-i} d \theta\right\} \leq a_{i}\right\} .
$$

Now define:

$$
V(\zeta)=\int_{\theta} \int_{a_{1}=0}^{a_{1}^{*}} \cdots \int_{a_{I}=0}^{a_{I}^{*}}\left(v(a)-v\left(a^{*}\right)\right) d F_{I}\left(\zeta_{I}\left(a_{I}\right)-\theta\right) \cdots d F_{1}\left(\zeta_{1}\left(a_{1}\right)-\theta\right) d \theta
$$

(The integrals are evaluated as sums if the action set is discrete.) Intuitively, $V(\zeta)$ is the expected value of $v(a)-v\left(a^{*}\right)$, conditional on $\theta \geq \theta^{*}-\frac{1}{2}$. The expectation is taken with respect to the improper prior, so this expression will only be well defined if each player eventually plays $a_{i}^{*}$, i.e., if $\zeta_{i}\left(a_{i}^{*}\right)$ is finite for all $i$. Otherwise $V(\zeta)$ will equal $-\infty$, since $v(a)<v\left(a^{*}\right)$ for all $a \neq a^{*}$.

Now consider the sequence $\zeta^{0}, \zeta^{1}, \ldots$, where $\zeta_{i}^{0}\left(a_{i}\right)=\theta^{*}$ for all $i$ and $a_{i} \in\left[0, a_{i}^{*}\right]$, and $\zeta^{n}=\beta\left(\zeta^{n-1}\right)$ for all $n>0$. An induction argument shows that this is an increasing sequence: $\zeta_{i}^{n}\left(a_{i}\right) \geq \zeta_{i}^{n-1}\left(a_{i}\right)$ for all $n, i$, and $a_{i}$. Moreover, $V\left(\zeta^{0}\right)$ is finite 
and negative. We will show that $V\left(\zeta^{n}\right)$ is increasing in $n$. Thus, $V\left(\lim _{n \rightarrow \infty} \zeta^{n}\right) \neq$ $-\infty$. This implies that in the limiting strategy profile $\lim _{n \rightarrow \infty} \zeta^{n}$, each player $i$ plays $a_{i}^{*}$ if her signal is high enough.

Let $\zeta=\zeta^{n}$ for any $n \geq 0$. Define $d F^{i}\left(a_{-i} \mid \theta\right)$ to be $d F_{I}\left(\zeta_{I}\left(a_{I}\right)-\theta\right) \cdots d F_{i+1}\left(\zeta_{i+1}\left(a_{i+1}\right)-\theta\right) d F_{i-1}\left(\beta(\zeta)_{i-1}\left(a_{i-1}\right)-\theta\right) \cdots d F_{1}\left(\beta(\zeta)_{1}\left(a_{1}\right)-\theta\right)$

This is the probability of the action profile $a_{-i}$ at the state $\theta$ if players $i-1$ and under play according to $\beta(\zeta)$ while players $i+1$ and above play according to $\zeta$. We separate $V(\beta(\zeta))-V(\zeta)$ into a telescopic sum and then integrate each summand by parts:

$$
\begin{aligned}
& V(\beta(\zeta))-V(\zeta)=\sum_{i=1}^{I} \iint_{\theta}\left\{\int_{a_{-i}}\left(v\left(a_{i}, a_{-i}\right)-v\left(a^{*}\right)\right) d\left[\begin{array}{c}
F_{i}\left(\beta(\zeta)_{i}\left(a_{i}\right)-\theta\right) \\
-F_{i}\left(\zeta_{i}\left(a_{i}\right)-\theta\right)
\end{array}\right]\right\} d F^{i}\left(a_{-i} \mid \theta\right) d \theta \\
& =\left.\sum_{i=1}^{I} \int_{\theta} \int_{a_{-i}}\left\{\left(v\left(a_{i}, a_{-i}\right)-v\left(a^{*}\right)\right)\left[\begin{array}{c}
F_{i}\left(\beta(\zeta)_{i}\left(a_{i}\right)-\theta\right) \\
-F_{i}\left(\zeta_{i}\left(a_{i}\right)-\theta\right)
\end{array}\right]\right\}\right|_{a_{i}=0} ^{a_{i}^{*}} d F^{i}\left(a_{-i} \mid \theta\right) d \theta \\
& -\sum_{i=1}^{I} \iint_{\theta}\left\{\int_{a_{-i}}\left\{\begin{array}{c}
F_{i}\left(\beta(\zeta)_{i}\left(a_{i}\right)-\theta\right) \\
-F_{i}\left(\zeta_{i}\left(a_{i}\right)-\theta\right)
\end{array}\right] \frac{\partial v\left(a_{i}, a_{-i}\right)}{\partial a_{i}} d a_{i}\right\} d F^{i}\left(a_{-i} \mid \theta\right) d \theta
\end{aligned}
$$

Since no action above $a_{i}^{*}$ is played in either strategy profile, $\beta(\zeta)_{i}\left(a_{i}\right)=\zeta_{i}\left(a_{i}\right)=$ $\infty$, so

$$
\left.\left\{\left(v\left(a_{i}, a_{-i}\right)-v\left(a^{*}\right)\right)\left[\begin{array}{c}
F_{i}\left(\beta(\zeta)_{i}\left(a_{i}\right)-\theta\right) \\
-F_{i}\left(\zeta_{i}\left(a_{i}\right)-\theta\right)
\end{array}\right]\right\}\right|_{a_{i}=0} ^{a_{i}^{*}}=-\left(v\left(0, a_{-i}\right)-v\left(a^{*}\right)\right)\left[\begin{array}{c}
F_{i}\left(\beta(\zeta)_{i}\left(a_{i}\right)-\theta\right) \\
-F_{i}\left(\zeta_{i}\left(a_{i}\right)-\theta\right)
\end{array}\right] \geq 0
$$

since $a^{*}$ maximizes $v$ and since $\beta(\zeta)_{i}(0) \geq \zeta_{i}(0)$. Thus,

$$
\begin{aligned}
& V(\beta(\zeta))-V(\zeta) \geq-\sum_{i=1}^{I} \iint_{\theta}\left\{\int_{a_{-i}} \int_{a_{i}=0}^{a_{i}^{*}}\left[\begin{array}{c}
F_{i}\left(\beta(\zeta)_{i}\left(a_{i}\right)-\theta\right) \\
-F_{i}\left(\zeta_{i}\left(a_{i}\right)-\theta\right)
\end{array}\right] \frac{\partial v\left(a_{i}, a_{-i}\right)}{\partial a_{i}} d a_{i}\right\} d F^{i}\left(a_{-i} \mid \theta\right) d \theta \\
& =-\sum_{i=1}^{I} \int_{a_{i}=0}^{a_{i}^{*}} \int_{\theta} \int_{a_{-i}} \frac{\partial v\left(a_{i}, a_{-i}\right)}{\partial a_{i}}\left[\begin{array}{c}
F_{i}\left(\beta(\zeta)_{i}\left(a_{i}\right)-\theta\right) \\
-F_{i}\left(\zeta_{i}\left(a_{i}\right)-\theta\right)
\end{array}\right] d F^{i}\left(a_{-i} \mid \theta\right) d \theta d a_{i}
\end{aligned}
$$


But for any $a_{i}$ and $\theta, F_{i}\left(\beta(\zeta)_{i}\left(a_{i}\right)-\theta\right)-F_{i}\left(\zeta_{i}\left(a_{i}\right)-\theta\right)$ is just the probability that a signal is observed between $\zeta_{i}\left(a_{i}\right)$ and $\beta(\zeta)_{i}\left(a_{i}\right)$ - the interval where under $\zeta$ player $i$ plays more than $a_{i}$ and her best response to $\zeta$ is to play something no greater than $a_{i}$. If instead players 1 through $i-1$ play according to $\beta(\zeta)$, player $i$ 's best response in this interval is still no greater than $a_{i}$ by strategic complementarities. Therefore, by own-action concavity, player $i$ 's payoff, conditional on getting a signal in this interval, must be weakly decreasing in her own action:

$$
\int_{\theta} \int_{a_{-i}} \frac{\partial g_{i}\left(a_{i}, a_{-i}\right)}{\partial a_{i}}\left[\begin{array}{c}
F_{i}\left(\bar{x}_{i}^{\beta(\zeta)}\left(a_{i}\right)-\theta\right) \\
-F_{i}\left(\bar{x}_{i}^{\zeta}\left(a_{i}\right)-\theta\right)
\end{array}\right] d F^{i}\left(a_{-i} \mid \theta\right) d \theta \leq 0
$$

Since $a_{i} \leq a_{i}^{*}, \frac{\partial v\left(a_{i}, a_{-i}\right)}{\partial a_{i}} \leq \mu_{i}\left(a_{i}\right) \frac{\partial g\left(a_{i}, a_{-i}\right)}{\partial a_{i}}$ by the LP-maximizer condition. Thus:

$$
\begin{aligned}
& V(\beta(\zeta))-V(\zeta) \\
& \geq-\sum_{i=1}^{I} \int_{a_{i}=0}^{a_{i}^{*}} \iint_{\theta} \frac{\partial v\left(a_{i}, a_{-i}\right)}{\partial a_{i}}\left[\begin{array}{c}
F_{i}\left(\bar{x}_{i}^{\beta(\zeta)}\left(a_{i}\right)-\theta\right) \\
-F_{i}\left(\bar{x}_{i}^{\zeta}\left(a_{i}\right)-\theta\right)
\end{array}\right] d F^{i}\left(a_{-i} \mid \theta\right) d \theta d a_{i} \\
& \geq-\sum_{i=1}^{I} \int_{a_{i}=0}^{a_{i}^{*}} \mu_{i}\left(a_{i}\right) \int_{\theta} \int_{a_{-i}} \frac{\partial g_{i}\left(a_{i}, a_{-i}\right)}{\partial a_{i}}\left[\begin{array}{c}
F_{i}\left(\bar{x}_{i}^{\beta(\zeta)}\left(a_{i}\right)-\theta\right) \\
-F_{i}\left(\bar{x}_{i}^{\zeta}\left(a_{i}\right)-\theta\right)
\end{array}\right] d F^{i}\left(a_{-i} \mid \theta\right) d \theta d a_{i} \\
& \geq 0
\end{aligned}
$$

as claimed.

This implies that for all $i$ and $a_{i} \in\left[0, a_{i}^{*}\right], \zeta_{i}^{n}\left(a_{i}\right)$ converges to some finite upper bound $\zeta_{i}^{\infty}\left(a_{i}\right)$ as $n$ grows. Let $\hat{s}^{1}$ be this upper bound written in standard notation (i.e., $\hat{s}_{i}^{1}\left(x_{i}\right)=\min \left\{a_{i}: \zeta_{i}^{\infty}\left(a_{i}\right) \geq x_{i}\right\}$ ). This is the smallest strategy profile surviving iterated deletion of strictly dominated strategies in $G^{* *}\left(1, \theta^{*}\right)$; moreover, there exists a $c>0$ such that $\hat{s}_{i}^{1}\left(x_{i}\right)=a_{i}^{*}$ for all $i$ and $x_{i} \geq \theta^{*}+c$.

Changing $\nu$ is equivalent to relabeling the game $G^{* *}\left(\nu, \theta^{*}\right)$. Thus if we write $\widehat{s}^{\nu}$ for the unique strategy profile surviving iterated deletion of strictly dominated strategies in $G^{* *}\left(\nu, \theta^{*}\right)$, we have $\widehat{s}^{\nu}\left(x_{i}\right)=\hat{s}^{1}\left(\frac{x_{i}-\theta^{*}}{\nu}\right)$. This in turn implies that $\widehat{s}_{i}^{\nu}\left(x_{i}\right)=a_{i}^{*}$ for all $x_{i} \geq \theta^{*}+\nu c$. 
But now if $s^{\nu}$ is the essentially unique equilibrium of $G^{*}(\nu)$, we have that $s^{\nu} \geq \widehat{s}^{\nu}$ (this is true because the game $G^{*}(\nu)$ has everywhere higher best responses than the game $\left.G^{* *}\left(\nu, \theta^{*}\right)\right)$. So we have:

Lemma 15 For all $\varepsilon>0$, there exists $\bar{\nu}$ such that for all $\nu \leq \bar{\nu}, s_{i}^{\nu}\left(\theta^{*}+\varepsilon\right) \geq a_{i}^{*}$.

A symmetric construction gives:

Lemma 16 For all $\varepsilon>0$, there exists $\bar{\nu}$ such that for all $\nu \leq \bar{\nu}, s_{i}^{\nu}\left(\theta^{*}-\varepsilon\right) \leq a_{i}^{*}$.

This proves the proposition. Q.E.D.

\subsection{Sufficient Conditions for an LP-Maximizer to Exist}

The LP-maximizer conditions of Definition 12 are rather complex. In Sections 5.1.1 and 5.1.2, we describe simpler conditions that are sufficient for an action profile to be an LP-maximizer. In Sections 5.1.3 to 5.1.5, we apply those results to give a complete characterization of the LP-maximizer in certain special classes of games.

\subsubsection{Weighted Potential Maximizers}

One sufficient condition for $a^{*}$ to be a local potential maximizer is that $a^{*}$ is a weighted potential maximizer. This is a slight generalization of Monderer and Shapley [1996]'s notion of an action profile that maximizes a potential function for a game.

Definition Action profile $a^{*}$ is a weighted potential maximizer (WP-maximizer) of $\mathbf{g}$ if there exists a vector $\mu \in \mathbf{R}_{+}^{I}$ and a weighted potential function $v: A \rightarrow \mathbf{R}$ with $v\left(a^{*}\right)>v(a)$ for all $a \neq a^{*}$, such that for all $i=1, . ., I, a_{i}, a_{i}^{\prime} \in A_{i}$ and $a_{-i} \in A_{-i}$,

$$
v\left(a_{i}, a_{-i}\right)-v\left(a_{i}^{\prime}, a_{-i}\right)=\mu_{i}\left[g_{i}\left(a_{i}, a_{-i}\right)-g_{i}\left(a_{i}^{\prime}, a_{-i}\right)\right] .
$$




\subsection{2 p-Dominance Conditions}

Let $\mathbf{p}=\left(p_{1}, \ldots, p_{I}\right)$. The notion of $\mathbf{p}$-dominance is a many player, many action game generalization of risk dominance (see Kajii and Morris [1997]). An action profile $a^{*}$ is $\mathbf{p}$-dominant if it is a best response for each player $i$ if she puts weight at least $p_{i}$ on her opponents' playing according to $a^{*}$ :

Definition $\mathbf{1 7}$ Action profile $a^{*}$ is $\mathbf{p}$-dominant in $g$ if

$$
\sum_{a_{-i}} \lambda_{i}\left(a_{-i}\right) g_{i}\left(a_{i}^{*}, a_{-i}\right) \geq \sum_{a_{-i}} \lambda_{i}\left(a_{-i}\right) g_{i}\left(a_{i}, a_{-i}\right),
$$

for all $i, a_{i} \in A_{i}$ and $\lambda_{i} \in \Delta\left(A_{-i}\right)$ with $\lambda_{i}\left(a_{-i}^{*}\right) \geq p_{i}$.

For low enough $\mathbf{p}, \mathbf{p}$-dominance is a sufficient condition for an action profile to be an LP-maximizer.

Lemma 18 If action profile $a^{*}$ is $p$-dominant for some $p$ with $\sum_{i=1}^{I} p_{i}<1$, then $a^{*}$ is an LP-maximizer.

Proof: see Morris [1999].

\subsubsection{Two Player, Two Action Games with Two Strict Nash Equilibria}

Let $I=2$ and $A_{1}=A_{2}=\{0,1\}$. Let $g_{1}(0,0)>g_{1}(1,0), g_{1}(1,1)>g_{1}(0,1)$, $g_{2}(0,0)>g_{2}(0,1)$ and $g_{2}(1,1)>g_{2}(1,0)$, so $(0,0)$ and $(1,1)$ are both strict Nash equilibria. Now let

$$
\begin{aligned}
q_{1}^{*} & =\frac{g_{1}(0,0)-g_{1}(1,0)}{g_{1}(0,0)-g_{1}(1,0)+g_{1}(1,1)-g_{1}(0,1)} \\
q_{2}^{*} & =\frac{g_{2}(0,0)-g_{2}(0,1)}{g_{2}(0,0)-g_{2}(0,1)+g_{2}(1,1)-g_{2}(1,0)}
\end{aligned}
$$

A weighted potential function $v$ is given by the following matrix:

\begin{tabular}{|l|l|l|}
\hline & 0 & 1 \\
\hline 0 & $q_{1}^{*}+q_{2}^{*}$ & $q_{1}^{*}$ \\
\hline 1 & $q_{2}^{*}$ & 1 \\
\hline
\end{tabular}


$(0,0)$ is a WP-maximizer if $q_{1}^{*}+q_{2}^{*}>1$ and $(1,1)$ is a WP-maximizer if $q_{1}^{*}+q_{2}^{*}<1$. Thus, generically, there is a WP-maximizer. The WP-maximizer is the risk dominant equilibrium in the sense of Harsanyi and Selten (1988).

\subsubsection{Many Player Two Action Games with Symmetric Payoffs}

Let $A_{i}=\{0,1\}$ and suppose $g_{i}\left(a_{i}, a_{-i}\right)=g\left(a_{i}, a_{-i}\right)$ depends only on $a_{i}$ and the number of players $j \neq i$ who play 1 . Let $\xi(n)$ be the relative payoff to playing 1 versus 0 when $n$ other players play 1 . (I.e., $\xi(n)=g\left(1, a_{-i}\right)-g\left(0, a_{-i}\right)$ for any $a_{-i}$ in which $n$ players play 1.) Assume strategic complementarities: i.e., $\xi(n)$ is increasing in $n$. Let the potential function be

$$
v(a)=\left\{\begin{array}{l}
\sum_{k=0}^{m-1} \xi(k), \text { if the number of players playing } 1 \text { in } a \text { is } m>0 \\
0, \text { if no players play } 1 \text { in } a
\end{array}\right.
$$

Also set $\mu_{i}=1$ for all $i$. One can easily verify that $\mathbf{1}=(1, . ., 1)$ is the WP-maximizer

if $\sum_{k=\mathbf{0}}^{I-1} \xi(k)>0$ and that $\mathbf{0}$ is the WP-maximizer if $\sum_{k=\mathbf{0}}^{I-1} \xi(k)<0$. Thus generically in this class of games, there exists a WP-maximizer.

An equivalent characterization of the WP-maximizer is the following. Suppose that a player had a uniform prior over the number of her opponents choosing action 1. If 1 is a best response to that conjecture, then $\mathbf{1}$ is the WP-maximizer; if 0 is a best response to that conjecture, then $\mathbf{0}$ is the WP-maximizer. These are equivalent since $\frac{1}{I} \sum_{k=0}^{I-1} \xi(k)$ is just the relative payoff to playing 1 if one has such a uniform prior.

\subsubsection{Two Player Three Action Games with Symmetric Payoffs}

Let $I=2, A_{1}=A_{2}=\{0,1,2\} ; g_{1}\left(a_{1}, a_{2}\right)=g_{2}\left(a_{2}, a_{1}\right)=w_{a_{1}} w_{a_{2}}$, where $w_{x x}>w_{y x}$ for all $y \neq x$ and $w_{x y}-w_{x^{\prime} y}>w_{x y^{\prime}}-w_{x^{\prime} y^{\prime}}$ if $x>x^{\prime}$ and $y>y^{\prime}$. Write $\Delta_{x^{\prime} y^{\prime}}^{x y}$ for the net expected gain of choosing action $x$ rather than $y$ against a $50 / 50$ conjecture on 
whether the opponent will choose action $x^{\prime}$ or $y^{\prime}$. Thus

$$
\Delta_{x^{\prime} y^{\prime}}^{x y}=w_{x^{\prime} x}+w_{x^{\prime} y}-w_{y^{\prime} x}-w_{y^{\prime} y} .
$$

Note that $\Delta_{x^{\prime} y^{\prime}}^{x y}=\Delta_{x^{\prime} y^{\prime}}^{y x}$ and $\Delta_{x^{\prime} y^{\prime}}^{x y}=-\Delta_{y^{\prime} x^{\prime}}^{x y}$. Note that $\Delta_{x y}^{x y}>0$ implies that action profile $(x, x)$ pairwise risk dominates action profile $(y, y)$. Now we have the following complete (for generic games) characterization of the LP-maximizers.

- $(0,0)$ is the LP-maximizer if $\Delta_{01}^{01}>0$ and either (1) $\Delta_{12}^{12}>0$ or (2) $\Delta_{21}^{21}>0$ and $\frac{\Delta_{10}^{02}}{\Delta_{01}^{01}}<\frac{\Delta_{12}^{02}}{\Delta_{21}^{21}}$.

- $(1,1)$ is the LP-maximizer if $\Delta_{10}^{10}>0$ and $\Delta_{12}^{12}>0$.

- $(2,2)$ is the LP-maximizer if $\Delta_{21}^{21}>0$ and either (1) $\Delta_{10}^{10}>0$ or (2) $\Delta_{01}^{01}>0$ and $\frac{\Delta_{10}^{02}}{\Delta_{01}^{01}}>\frac{\Delta_{12}^{02}}{\Delta_{21}^{21}}$.

The following example illustrates these conditions:

\begin{tabular}{|l|l|l|l|}
\hline$\left(g_{1}, g_{2}\right)$ & 0 & 1 & 2 \\
\hline 0 & 4,4 & 0,0 & $-6,-3$ \\
\hline 1 & 0,0 & 1,1 & 0,0 \\
\hline 2 & $-3,-6$ & 0,0 & 2,2 \\
\hline
\end{tabular}

$(0,0)$ is the LP-maximizer, since $\Delta_{01}^{01}=3, \Delta_{21}^{21}=1, \Delta_{10}^{02}=2$ and $\Delta_{12}^{02}=1$. Note that $(2,2)$ pairwise risk dominates both $(1,1)$ and $(0,0)$, but nonetheless is not the LP-maximizer.

Proving the above claims (i.e., constructing the local potential functions) involves tedious algebra. Here, we will just note two cases to illustrate the issues.

Case 1: $\Delta_{10}^{10}>0$ and $\Delta_{12}^{12}>0$. Consider the following local potential function:

\begin{tabular}{|l|l|l|l|}
\hline$v$ & 0 & 1 & 2 \\
\hline 0 & $-\Delta_{10}^{10}$ & $w_{01}-w_{11}$ & $-\varepsilon$ \\
\hline 1 & $w_{01}-w_{11}$ & 0 & $w_{21}-w_{11}$ \\
\hline 2 & $-\varepsilon$ & $w_{21}-w_{11}$ & $-\Delta_{12}^{12}$ \\
\hline
\end{tabular}


for some small but strictly positive $\varepsilon$. Setting $a^{*}=(1,1)$ and $\mu_{1}(0)=\mu_{1}(2)=$ $\mu_{2}(0)=\mu_{2}(2)=1$, one can verify that the inequalities of equation (1) are satisfied.

Case 2: $\Delta_{01}^{01}>0, \Delta_{21}^{21}>0, \Delta_{10}^{02}>0, \Delta_{12}^{02}>0$ and $\frac{\Delta_{10}^{02}}{\Delta_{01}^{01}}<\frac{\Delta_{12}^{02}}{\Delta_{21}^{21}}$. Consider the following local potential function:

\begin{tabular}{|l|l|l|l|}
\hline$v$ & 0 & 1 & 2 \\
\hline 0 & $\varepsilon$ & $\varepsilon+\lambda_{1}[w(1,0)-w(0,0)]$ & $\lambda_{1}\left[w_{\mathbf{0} 2}-w_{12}\right]+\lambda_{2}\left[w_{12}-w_{22}\right]$ \\
\hline 1 & $\varepsilon+\lambda_{1}\left[w_{10}-w_{\mathbf{0 0}}\right]$ & $-\lambda_{2} \Delta_{21}^{21}$ & $\lambda_{2}\left[w_{12}-w_{22}\right]$ \\
\hline 2 & $\lambda_{1}\left[w_{\mathbf{0} 2}-w_{12}\right]+\lambda_{2}\left[w_{12}-w_{22}\right]$ & $\lambda_{2}\left[w_{12}-w_{22}\right]$ & 0 \\
\hline
\end{tabular}

for some small but strictly positive $\varepsilon$ and positive $\lambda_{1}$ and $\lambda_{2}$ such that

$$
\frac{\Delta_{21}^{21}}{\Delta_{01}^{01}}<\frac{\lambda_{1}}{\lambda_{2}}<\frac{\Delta_{12}^{02}}{\Delta_{10}^{02}} .
$$

Setting $a^{*}=(0,0), \mu_{1}(1)=\mu_{2}(1)=\lambda_{1}, \mu_{1}(2)=\mu_{2}(2)=\lambda_{2}$, one can verify that the inequalities of equation (1) are satisfied.

\section{Conclusion}

For two player, two action games, Carlsson and van Damme [1993a] showed that a unique equilibrium is played as the noise in the payoff signals goes to zero. They also showed that the equilibrium selected is independent of the structure of the noise. In the language of this paper, they showed both a limit uniqueness and a noise independent selection result. One contribution of this paper is to identify sufficient conditions under which both results generalize to many player, many action games. In doing so, we generalize a number of papers in the literature. For many player, two action games, Carlsson and van Damme [1993b], Kim [1996], and Morris and Shin $[1998,1999]$ showed limit uniqueness results, for particular assumptions about the structure of the noise. ${ }^{1}$ Our results here show both limit uniqueness and noise

\footnotetext{
${ }^{1}$ Carlsson and van Damme [1993b], Kim [1996] and Morris and Shin [1998] assume uniform noise distributions, while Morris and Shin [1999] assume normally distributed noise. In fact, the
} 
independent selection results that do not depend on the shape of the noise. Carlsson and Ganslandt [1998] show limit uniqueness and noise independent selection results for a minimum effort coordination game. Since theirs is a potential game, these results too are special cases of the analysis of this paper. Our general sufficient condition for noise independent selection (own-action concavity and the local potential maximizer condition) also works in settings where there are no existing results (e.g., two player three action games).

We also showed by example the limits to noise independent selection. Our example in which noise independent selection fails is for a two player, four action symmetric payoff game. This example is minimal in the sense that noise independent selection must hold with two players and symmetric payoffs if there are fewer than four actions. Carlsson [1989] gives a three player, two action example in which noise independent selection failed.

Our analysis emphasizes the distinction between limit uniqueness and noise independent selection. We are able to show a quite general limit uniqueness result by combining the local noise structure of Carlsson and van Damme [1993a] with the appropriate monotonicity properties for a many player, many action game. But noise independent selection imposes much more structure on the payoffs on the game. In this sense, it is easy to get limit uniqueness without noise independent selection.

Our noise independent selection results are related to work on the robustness of equilibria to incomplete information (Kajii and Morris [1997]). A Nash equilibrium of a complete information game is robust to incomplete information if every incomplete information game in which payoffs are almost always given by that complete

\footnotetext{
continuum player assumption in Morris and Shin [1998, 1999] and the unbounded support of the noise distribution in Morris and Shin [1999] are not consistent with the model of this paper; however, these differences affect neither the limit uniqueness result nor the noise independent selection. (There was a mistake in the characterization of the noise independent selection in Morris and Shin [1998]; the corrected version in Heinemann [1999] is consistent with the characterization in this paper).
} 
information game has an equilibrium in which that Nash equilibrium is almost always played. Kajii and Morris showed that risk dominant equilibria of two player, two action games and, more generally, $p$-dominant equilibria of many player, many

action games with $\sum_{i=1}^{I} p_{i}<1$ are robust to incomplete information. The global games introduced by Carlsson and van Damme [1993a] and studied in this paper represent a different way of adding an intuitively small amount of incomplete information about payoffs. However, one can show that if a complete information game has a robust equilibrium, then that equilibrium must be the noise independent selection. Thus, the sufficient conditions for noise independent selection in this paper are in fact also sufficient for robustness to incomplete information (see Morris [1999]).

Our limit uniqueness argument exploits the stationarity of the distribution of signal differences in the limit as the noise shrinks. We show that multiple equilibria would require that there be two agents who want to play the same action (or, with discrete actions, who are indifferent between the same two actions) and who face the same distribution of opponents' actions, yet who see different signals. Since this is impossible, the equilibrium is unique. A closely related argument underlies limit uniqueness results for dynamic games with complete information and frictions in changing actions (Burdzy, Frankel, and Pauzner [1999]; Frankel [2000]; Frankel and Pauzner [1999a,b]). In such games, payoff shocks also give rise to a unique equilibrium. While the present paper relies on the stationarity of signal differences, the cited papers use the stationarity of the payoff shocks to prove an analogous result. ${ }^{2}$

\section{Appendix}

Proof of LEMMA 2. Define $B R_{i}\left(s, x_{i}\right)$ to be the set of optimal actions for a player

\footnotetext{
${ }^{2}$ Frankel [2000] extends this argument to the case of general nonstationary payoff shocks. He shows that space and time can be transformed so that the payoff shocks are stationary in the new coordinates, whereupon the prior argument can be applied.
} 
$i$ who sees signal $x_{i}$ and whose opponents play according to the strategy $s$ :

$$
B R_{i}\left(s, x_{i}\right)=\underset{a_{i} \in A_{i}}{\arg \max } \int_{z \in R^{I-1}} \pi_{i}(z) u_{i}\left(\left(a_{i},\left(s_{j}^{k}\left(x_{i}+\nu z_{j}\right)\right)_{j \neq i}\right), x_{i}\right) d z
$$

where

$$
\pi_{i}(z) \equiv \int_{\theta^{\prime}=-\infty}^{\infty} f_{i}\left(-\theta^{\prime}\right) \prod_{j \neq i} f_{j}\left(z_{j}-\theta^{\prime}\right) d \theta^{\prime}
$$

Note that by $\mathbf{A} 4$ and $\mathbf{A} 5$ and the theorem of the maximum, $B R_{i}$ must be upperhemi-continuous in $x_{i}$, even if $s$ is discontinuous. (Because of the noise in the signals, $i$ 's posterior over the distribution of opponents' actions changes continuously in $x_{i}$ ).

We first do iterated dominance to establish a lower bound on the actions players can choose for each signal. We define a sequence of strategy profiles $s^{k} \equiv\left(s_{1}^{k}, \ldots, s_{I}^{k}\right)$ for $k=0,1, \ldots$ as follows. Let $s_{i}^{0}\left(x_{i}\right)=0$ for all $x_{i} \in \mathbf{R}$ and let $s^{k+1}$ be the smallest best response to $s^{k}: s_{i}^{k+1}\left(x_{i}\right)=\min B R_{i}\left(s^{k}, x_{i}\right)$. A1 and $\mathbf{A} \mathbf{3}$ imply that (i) $s_{i}^{k}\left(x_{i}\right)$ is weakly increasing in $x_{i}$ for all $(i, k)$ and (ii) $s_{i}^{k}\left(x_{i}\right)$ is weakly increasing in $k$ for all $\left(i, x_{i}\right)$. A2 implies $s_{i}^{k}(\underline{\theta})=0$ and $s_{i}^{k}(\bar{\theta})=1$ for all $i$ and for $k \geq 1$. Let $\underline{s}$ be defined by $\underline{s}_{i}\left(x_{i}\right)=\lim _{k \rightarrow \infty} s_{i}^{k}\left(x_{i}\right)$. A player $i$ seeing signal $x_{i}$ must choose an action that is at least $\underline{s}_{i}\left(x_{i}\right)$. By induction, $\underline{s}_{i}$ is weakly increasing. Since $B R_{i}$ is upper hemicontinuous and (by an induction argument) $\min B R_{i}\left(\underline{s}, x_{i}\right)$ is weakly increasing in $x_{i}, \underline{s}_{i}\left(x_{i}\right)$ must be left continuous.

We next construct an upper bound on players' actions. For any $\lambda \in \Re$, let $s^{\lambda}=\left(s_{1}^{\lambda}, \ldots, s_{I}^{\lambda}\right)$ denote the translation of $\underline{s}$ to the left by $\lambda$ : for all $i$ and $x_{i}$, $s_{i}^{\lambda}\left(x_{i}\right)=\underline{s}_{i}\left(x_{i}+\lambda\right)$. Let $\lambda_{0}$ be large enough that, for all $i, s_{i}^{\lambda_{0}}(\underline{\theta})=1$; since $s^{\lambda_{0}}$ is weakly increasing, a player $i$ with any signal $x_{i}$ will never play an action that is greater than $s_{i}^{\lambda_{0}}\left(x_{i}\right)$. Now let $\lambda_{k}$ be the smallest number such that no player $i$ who expects others to play according to $s^{\lambda_{k-1}}$ will ever play above $s_{i}^{\lambda_{k}}$; more precisely,

$$
\lambda_{k}=\inf \left\{\lambda: s_{i}^{\lambda}\left(x_{i}\right) \geq \max B R_{i}\left(s^{\lambda_{k-1}}, x_{i}\right) \forall i, x_{i}\right\}
$$

A1 and A3 imply that $\lambda_{k}$ is weakly decreasing in $k$. Let $\lambda_{\infty}=\lim _{k \rightarrow \infty} \lambda_{k}$, and denote $\bar{s}=s^{\lambda_{\infty}}$. (Note that $\lambda_{\infty} \geq 0$ since the iterations cannot go beyond $\underline{s}$.) 
By construction, a player $i$ who sees signal $x_{i}$ will never play an action that exceeds the right continuous version of $\bar{s}_{i}: \lim _{z \downarrow} \bar{x}_{i} \bar{s}_{i}(z) \geq \max B R_{i}\left(\bar{s}, x_{i}\right)$ for all $x_{i}$. We will show that by contradiction that $\lambda_{\infty}=0: \bar{s}$ and $\underline{s}$ coincide. This means that any equilibrium must be a weakly increasing function that agrees with $\underline{s}$ at all points of continuity. This will prove the lemma since a monotonic function can have at most a countable number of discontinuities.

Claim 1 There is a player $i$ and a signal $x_{i}^{*}$ such that $\bar{s}_{i}\left(x_{i}^{*}-\varepsilon\right)<\max B R_{i}\left(\bar{s}, x_{i}^{*}+\varepsilon\right)$ for all $\varepsilon>0$.

Proof Since $\bar{s}$ is the limit of the iterations from the left, for all $\varepsilon>0$ there is a player $i$ and a signal $x_{i}$ such that $\bar{s}_{i}\left(x_{i}-\varepsilon\right)<\max B R_{i}\left(\bar{s}, x_{i}\right)$. (Otherwise let $\varepsilon^{\prime}$ be such that there is no $i$ and $x_{i}$ for which $\bar{s}_{i}\left(x_{i}-\varepsilon^{\prime}\right)<\max B R_{i}\left(\bar{s}, x_{i}\right)$; define $\lambda_{\infty}^{\prime}=\lambda_{\infty}-\varepsilon^{\prime}$. The limit of the iterations must be no greater than $\lambda_{\infty}^{\prime}$, a contradiction.) Since the number of players is finite and each $\bar{s}_{i}$ is weakly increasing, there must be a particular player $i$ such that for all $\varepsilon>0, \bar{s}_{i}\left(x_{i}(\varepsilon)-\varepsilon\right)<\max B R_{i}\left(\bar{s}, x_{i}(\varepsilon)\right)$ for some $x_{i}(\varepsilon)$. Define $x_{i}^{\prime}(\varepsilon)=x_{i}(2 \varepsilon)-\varepsilon$; we know that for all $\varepsilon>0, \bar{s}_{i}\left(x_{i}^{\prime}(\varepsilon)-\varepsilon\right)<\max B R_{i}\left(\bar{s}, x_{i}^{\prime}(\varepsilon)+\varepsilon\right)$. Since for all $\varepsilon, x_{i}^{\prime}(\varepsilon) \in[\underline{\theta}, \bar{\theta}]$, there is a convergent subsequence of $x_{i}^{\prime}(\varepsilon)$ as $\varepsilon \rightarrow 0$; let $x_{i}^{*}$ be the limit. For all $\varepsilon>0, \bar{s}_{i}\left(x_{i}^{*}-\varepsilon\right)<\max B R_{i}\left(\bar{s}, x_{i}^{*}+\varepsilon\right)$. (Why: we can take $\varepsilon^{\prime}$ small enough that $\bar{s}_{i}\left(x_{i}^{\prime}\left(\varepsilon^{\prime}\right)-\varepsilon / 2\right)<\max B R_{i}\left(\bar{s}, x_{i}^{\prime}\left(\varepsilon^{\prime}\right)+\varepsilon / 2\right)$ and $\left|x_{i}^{\prime}\left(\varepsilon^{\prime}\right)-x_{i}^{*}\right|<\varepsilon / 2$; hence, $\bar{s}_{i}\left(x_{i}^{\prime}\left(\varepsilon^{\prime}\right)-\varepsilon / 2\right) \geq \bar{s}_{i}\left(x_{i}^{*}-\varepsilon\right)$ and $\max B R_{i}\left(\bar{s}, x_{i}^{\prime}\left(\varepsilon^{\prime}\right)+\varepsilon / 2\right) \leq \max B R_{i}\left(\bar{s}, x_{i}^{*}+\varepsilon\right)$.) Q.E.D.(Claim 1)

Claim 2 If $\lambda_{\infty}>0$ then $\max B R_{i}\left(\bar{s}, x_{i}^{*}\right)=\bar{s}_{i}\left(x_{i}^{*}\right)$.

Proof Consider a player A receiving the signal $x_{i}^{*}+\lambda_{\infty}$, who believes that the other players play according to $\underline{s}$. By construction, she must be willing to play the action $a=\underline{s}_{i}\left(x_{i}^{*}+\lambda_{\infty}\right)$, i.e., $a \in B R_{i}\left(\underline{s}, x_{i}^{*}+\lambda_{\infty}\right)$. By A3, a player B receiving the signal $x_{i}^{*}$, who believes that the other players play according to $\bar{s}$ (and who thus expects the same distribution of opponents' actions as player A) cannot be willing to play an 
action $a^{\prime}>a$ : since $\lambda_{\infty}>0$, this would imply that player A strictly prefers $a^{\prime}$ to $a$, a contradiction. This shows that $\max B R_{i}\left(\bar{s}, x_{i}^{*}\right) \leq a=\bar{s}_{i}\left(x_{i}^{*}\right)$. Moreover, since $B R_{i}$ is upper hemicontinuous and $\max B R_{i}\left(\bar{s}, x_{i}\right)$ is weakly increasing in $x_{i}$, max $B R_{i}\left(\bar{s}, x_{i}\right)$ is right continuous in $x_{i}$. Hence, $\max B R_{i}\left(\bar{s}, x_{i}^{*}\right)=\lim _{\varepsilon_{\downarrow} \mathbf{0}} \max B R_{i}\left(\bar{s}, x_{i}^{*}+\varepsilon\right) \geq$ $\lim _{\varepsilon \downarrow \mathbf{0}} \bar{s}_{i}\left(x_{i}^{*}-\varepsilon\right)=\bar{s}_{i}\left(x_{i}^{*}\right)$. So $\max B R_{i}\left(\bar{s}, x_{i}^{*}\right)=\bar{s}_{i}\left(x_{i}^{*}\right)$. Q.E.D.(Claim 2)

Since $\bar{s}_{i}\left(x_{i}^{*}-\varepsilon\right)<\max B R_{i}\left(\bar{s}, x_{i}^{*}+\varepsilon\right)$ for all $\varepsilon>0$ and $\bar{s}_{i}\left(x_{i}^{*}\right)=\max B R_{i}\left(\bar{s}, x_{i}^{*}\right)$, and since both $\bar{s}_{i}\left(x_{i}\right)$ and $\max B R_{i}\left(\bar{s}, x_{i}\right)$ are weakly increasing in $x_{i}$, there are two possibilities: either

(a) for all $\varepsilon>0, \max B R_{i}\left(\bar{s}, x_{i}^{*}+\varepsilon\right)>\max B R_{i}\left(\bar{s}, x_{i}^{*}\right)$, or

(b) for all $\varepsilon>0, \bar{s}_{i}\left(x_{i}^{*}-\varepsilon\right)<\bar{s}_{i}\left(x_{i}^{*}\right)$.

We will show that each is impossible.

First, (a) implies that $\min B R_{i}\left(\bar{s}, x_{i}^{*}+\varepsilon\right)>\max B R_{i}\left(\bar{s}, x_{i}^{*}\right)$ for all $\varepsilon>0 .^{3}$ We claim that $\min B R_{i}\left(\underline{s}, x_{i}^{*}+\lambda_{\infty}\right) \geq \min B R_{i}\left(\bar{s}, x_{i}^{*}+\varepsilon\right)$ for small enough $\varepsilon$. The intuition is that a player facing strategy $\underline{s}$ and getting signal $x_{i}^{*}+\lambda_{\infty}$ has a strictly higher payoff parameter than someone facing strategy $\bar{s}$ and getting signal $x_{i}^{*}+\varepsilon$, but the action distribution they face converges to that faced by the second player as $\varepsilon \rightarrow 0$. To show this, it is enough to prove:

(*) For all $a_{i}$ and $a_{i}^{\prime}$,

$$
A=\int_{z \in \Re^{I-1}} \pi_{i}(z) \Delta u_{i}\left(a_{i}, a_{i}^{\prime}, \bar{s}_{j}\left(x_{i}^{*}+\varepsilon+\nu z_{j}\right)_{j \neq i}, x_{i}^{*}+\varepsilon\right) d z
$$

is no greater than

$$
B=\int_{z \in \Re^{I-1}} \pi_{i}(z) \Delta u_{i}\left(\left(a_{i}, a_{i}^{\prime}, \underline{s}_{j}\left(x_{i}^{*}+\lambda_{\infty}+\nu z_{j}\right)_{j \neq i}\right), x_{i}^{*}+\lambda_{\infty}\right) d z
$$

${ }^{3}$ Otherwise, suppose that $\varepsilon^{\prime}>0$ is such that $a=\min B R_{i}\left(\bar{s}, x_{i}^{*}+\varepsilon^{\prime}\right) \leq \max B R_{i}\left(\bar{s}, x_{i}^{*}\right)$. Then by A3, player $i$ strictly prefers action $a$ to any higher action if she observes any signal $x_{i}^{*}+\varepsilon$ that is lower than $x_{i}^{*}+\varepsilon^{\prime}$. This contradicts condition (a). 
Since by definition $\bar{s}_{j}\left(x_{j}\right)=\underline{s}_{j}\left(x_{j}+\lambda_{\infty}\right)$,

$$
\begin{aligned}
B & =\int_{z \in \Re^{I-1}} \pi_{i}(z) \Delta u_{i}\left(\left(a_{i}, a_{i}^{\prime}, \bar{s}_{j}\left(x_{i}^{*}+\nu z_{j}\right)_{j \neq i}\right), x_{i}^{*}+\lambda_{\infty}\right) d z \\
& =A+\int_{z \in \Re^{I-1}} \pi_{i}(z)\left[\begin{array}{c}
\Delta u_{i}\left(\left(a_{i}, a_{i}^{\prime}, \bar{s}_{j}\left(x_{i}^{*}+\varepsilon+\nu z_{j}\right)_{j \neq i}\right), x_{i}^{*}+\lambda_{\infty}\right) \\
-\Delta u_{i}\left(\left(a_{i}, a_{i}^{\prime}, \bar{s}_{j}\left(x_{i}^{*}+\varepsilon+\nu z_{j}\right)_{j \neq i}\right), x_{i}^{*}+\varepsilon\right)
\end{array}\right] d z \\
& -\int_{z \in \Re^{I-1}} \pi_{i}(z)\left[\begin{array}{c}
\Delta u_{i}\left(\left(a_{i}, a_{i}^{\prime}, \bar{s}_{j}\left(x_{i}^{*}+\varepsilon+\nu z_{j}\right)_{j \neq i}\right), x_{i}^{*}+\lambda_{\infty}\right) \\
-\Delta u_{i}\left(\left(a_{i}, a_{i}^{\prime}, \bar{s}_{j}\left(x_{i}^{*}+\nu z_{j}\right)_{j \neq i}\right), x_{i}^{*}+\lambda_{\infty}\right)
\end{array}\right] d z
\end{aligned}
$$

Consider the last expression, the sum of three terms. By A3 there is a constant $K$ such that the middle term in this expression is at least $K\left(\lambda_{\infty}-\varepsilon\right)\left(a_{i}-a_{i}^{\prime}\right){ }^{4}$ This approaches a strictly positive limit as $\varepsilon$ shrinks.

By A6, there is a constant $K^{\prime}$ such that the last term in (4) is no greater than

$$
\begin{aligned}
& \int_{z \in \Re^{I-1}} \pi_{i}(z)\left[K^{\prime}\left(a_{i}-a_{i}^{\prime}\right) \sum_{j \neq i}\left(\bar{s}_{j}\left(x_{i}^{*}+\varepsilon+\nu z_{j}\right)-\bar{s}_{j}\left(x_{i}^{*}+\nu z_{j}\right)\right)\right] d z \\
& =K^{\prime}\left(a_{i}-a_{i}^{\prime}\right) \sum_{j \neq i} \int_{z \in \Re^{I-1}} \pi_{i}(z)\left(\bar{s}_{j}\left(x_{i}^{*}+\varepsilon+\nu z_{j}\right)-\bar{s}_{j}\left(x_{i}^{*}+\nu z_{j}\right)\right) d z
\end{aligned}
$$

Because each $\bar{s}_{j}$ is bounded and continuous almost everywhere, each integral in this sum converges to zero as $\varepsilon$ shrinks (by the bounded convergence theorem), so the sum does as well. So the sum of the last two terms in (4) converges to a strictly positive limit as $\varepsilon$ shrinks. This proves $\left(^{*}\right)$. Hence, $\bar{s}_{i}\left(x_{i}^{*}\right)=\underline{s}_{i}\left(x_{i}^{*}+\lambda_{\infty}\right) \geq \min B R_{i}\left(\underline{s}, x_{i}^{*}+\right.$ $\left.\lambda_{\infty}\right)>\max B R_{i}\left(\bar{s}, x_{i}^{*}\right)$, contradicting Claim 2.

${ }^{4}$ With a continuous action space, let

$$
K=\min _{a, y \in[0,1]^{I} \times\left[x_{i}^{*}+\varepsilon, x_{i}^{*}+\lambda_{\infty}\right]} \frac{\partial^{2} u_{i}(a, y)}{\partial a_{i} \partial y}>0 ;
$$

with a discrete action space, let

$$
K=\min _{a_{i}, a_{i}^{\prime}, a_{-i}} \frac{\Delta u_{i}\left(a_{i}, a_{i}^{\prime}, a_{-i}, x_{i}^{*}+\lambda_{\infty}\right)-\Delta u_{i}\left(a_{i}, a_{i}^{\prime}, a_{-i}, x_{i}^{*}+\varepsilon\right)}{\left(a_{i}-a_{i}^{\prime}\right)\left(\lambda_{\infty}-\varepsilon\right)}>0 .
$$


Condition (b) implies that for all $\varepsilon>0, \underline{s}_{i}\left(x_{i}^{*}-\varepsilon+\lambda_{\infty}\right)<\underline{s}_{i}\left(x_{i}^{*}+\lambda_{\infty}\right)$. A player facing strategy $\bar{s}$ and getting signal $x_{i}^{*}$ has a strictly lower payoff parameter than someone facing strategy $\underline{s}$ and getting signal $x_{i}^{*}-\varepsilon+\lambda_{\infty}$ for small enough $\varepsilon$, but the action distribution they face converges to that faced by the second player as $\varepsilon \rightarrow 0$. Hence, by an analogous argument to that given in the preceding paragraph, they cannot want to play a higher action than someone facing strategy $\underline{s}$ and getting signal $x_{i}^{*}-\varepsilon+\lambda_{\infty}$ if $\varepsilon$ is sufficiently small. Thus, for small enough $\varepsilon, \bar{s}_{i}\left(x_{i}^{*}\right) \leq$ $\underline{s}_{i}\left(x_{i}^{*}-\varepsilon+\lambda_{\infty}\right)<\underline{s}_{i}\left(x_{i}^{*}+\lambda_{\infty}\right)$, which is impossible.

Since neither (a) nor (b) can hold, $\lambda_{\infty}$ must equal 0.

When the action set is finite, assumption $\mathbf{A} \mathbf{2}$ can be replaced by $\mathbf{A} \mathbf{2}^{\prime}$. The beginning of the proof must be modified as follows. Let $\underline{a}$ and $\bar{a}$ be the unique Nash equilibrium strategy profiles for $\theta<\underline{\theta}$ and for $\theta>\bar{\theta}$, respectively. For any positive integer $n$, consider the vector $x$ given by $x_{i}=\underline{\theta}-n \nu$ for all $i$. Since $\underline{a}$ is the unique Nash equilibrium for the payoff vector $x$, the only way that the players can play something other than $\underline{a}$ is if some player $j$ is uncertain (under $\underline{s}$ ) about some other player's action: for some $i \neq j, \underline{s}_{i}$ must take on more than one value for signals $x_{i} \in[\underline{\theta}-(n+1) \nu, \underline{\theta}-(n-1) \nu] .{ }^{5}$ Since $\underline{s}_{i}$ is monotonic and $A_{i}$ is finite, this condition can hold for at most a finite set of positive integers $n$. In particular, there is a $\underline{\theta}^{\prime}<\underline{\theta}$ below which $\underline{a}$ must be played: for all $i$, if $x_{i}<\underline{\theta}^{\prime}=\underline{\theta}-\nu \sum_{i=1}^{I}\left|A_{i}\right|$ then $\underline{s}_{i}(\theta)=\underline{a}_{i}$. Likewise, $\bar{a}$ must be played for signals above $\bar{\theta}^{\prime}=\bar{\theta}+\nu \sum_{i=1}^{I}\left|A_{i}\right|$.

We now iterate from above to obtain an upper bound on the set of equilibrium strategies. For all $i$, let $\hat{s}_{i}^{0}\left(x_{i}\right)=1$ for all $x_{i} \in \mathbf{R}$ and let $s_{i}^{k+1}\left(x_{i}\right)=\max B R_{i}\left(s^{k}, x_{i}\right)$. Let $\widehat{s}$ be defined by $\widehat{s}_{i}\left(x_{i}\right)=\lim _{k \rightarrow \infty} \hat{s}_{i}^{k}\left(x_{i}\right)$. A player $i$ seeing signal $x_{i}$ must choose an action that is at most $\hat{s}_{i}\left(x_{i}\right)$. By induction, $\hat{s}_{i}$ is weakly increasing. The preceding argument implies that under $\widehat{s}$, players must play $\underline{a}$ for signals below $\underline{\theta^{\prime}}$ and $\bar{a}$ for signals above $\bar{\theta}^{\prime}$.

\footnotetext{
${ }^{5}$ Because of the bounded supports, player $j$ knows that all other players' signals will be within $\nu$ of $x_{j}=\underline{\theta}-n \nu$.
} 
For any $\lambda \in \Re$, let $s^{\lambda}=\left(s_{1}^{\lambda}, \ldots, s_{I}^{\lambda}\right)$ denote the translation of $\underline{s}$ to the left by $\lambda$. Let $\lambda_{\mathbf{0}}$ be large enough that, for all $i$ and signals $x_{i}, s_{i}^{\lambda_{0}}\left(x_{i}\right) \geq \hat{s}_{i}\left(x_{i}\right) . \quad\left(\lambda_{0}=\bar{\theta}^{\prime}-\underline{\theta}^{\prime}\right.$ will suffice.) Players cannot choose actions that lie above $s^{\lambda_{0}}$. The rest of the proof proceeds as before: we iterate from the left using translations $s^{\lambda_{k}}$ until we reach a limit, and prove (using identical arguments) that this limit must equal $\underline{s}$. Q.E.D.Lemma 2

Proof of LEMMA 3. Denote

$$
\rho(\nu)=\max _{i=1, . ., I} \sup _{x_{i} \in \mathbf{B}} \frac{\max _{\theta^{\prime} \in\left[-\frac{1}{2}, \frac{1}{2}\right]} \phi\left(x_{i}+\nu \theta^{\prime}\right)}{\min _{\theta^{\prime} \in\left[-\frac{1}{2}, \frac{1}{2}\right]} \phi\left(x_{i}+\nu \theta^{\prime}\right)} .
$$

Let $b<\infty$ be such that $B \subset[-b+1, b-1]$. Since $\phi$ is positive, it has a positive minimum over the compact interval $[-b, b]$. Since it is also continuous, $\max _{\theta^{\prime} \in\left[-\frac{1}{2}, \frac{1}{2}\right]} \phi\left(x_{i}+\nu \theta^{\prime}\right)-$ $\min _{\theta^{\prime} \in\left[-\frac{1}{2}, \frac{1}{2}\right]} \phi\left(x_{i}+\nu \theta^{\prime}\right)$ converges to 0 as $\nu \rightarrow 0$ uniformly for $x_{i} \in[-b, b]$. Hence,

$$
\rho(\nu)=\max _{i=1, . ., I} \sup _{[-b, b]} 1+\frac{\max _{\theta^{\prime} \in\left[-\frac{1}{2}, \frac{1}{2}\right]} \phi\left(x_{i}+\nu \theta^{\prime}\right)-\min _{\theta^{\prime} \in\left[-\frac{1}{2}, \frac{1}{2}\right]} \phi\left(x_{i}+\nu \theta^{\prime}\right)}{\min _{\theta^{\prime} \in\left[-\frac{1}{2}, \frac{1}{2}\right]} \phi\left(x_{i}+\nu \theta^{\prime}\right)} \rightarrow 1 \text { as } \nu \rightarrow 0 .
$$

Since the support of each $f_{i}$ is contained in the interval $\left[-\frac{1}{2}, \frac{1}{2}\right]$, for all $x_{i} \in \mathbf{B}$,

$$
\frac{\pi_{i}(z)}{\rho(\nu)} \leq \tilde{\pi}_{i}\left(z \mid x_{i}, \nu\right) \leq \rho(\nu) \pi_{i}(z) .
$$

since

$$
\begin{aligned}
& \tilde{\pi}_{i}\left(z \mid x_{i}, \nu\right)=\frac{\int_{\theta=-\infty}^{\infty} \phi(\theta) f_{i}\left(\frac{x_{i}-\theta}{\nu}\right) \prod_{j \neq i} f_{j}\left(\frac{x_{j}-\theta}{\nu}\right) d \theta}{\int_{\theta=-\infty}^{\infty} \phi(\theta) f_{i}\left(\frac{x_{i}-\theta}{\nu}\right) d \theta} \\
&= \int_{\theta^{\prime}=-\infty}^{\infty} \phi\left(x_{i}+\nu \theta^{\prime}\right) f_{i}\left(-\theta^{\prime}\right) \prod_{j \neq i} f_{j}\left(z_{j}-\theta^{\prime}\right) d \theta^{\prime} \\
& \theta^{\prime}=-\infty
\end{aligned}
$$


Thus, for any event $E \subseteq \mathbf{R}^{I-1}$,

$$
\begin{aligned}
\left|\int_{k \in E} \tilde{\pi}_{i}\left(z \mid x_{i}, \nu\right) d z-\int_{z \in E} \pi_{i}(z) d z\right| & \leq\left(\int_{\ell \in E} \pi_{i}(z) d z\right) \max \left(\rho(\nu)-1, \frac{1}{\rho(\nu)}-1\right) \\
& \leq \max \left(\rho(\nu)-1, \frac{1}{\rho(\nu)}-1\right) .
\end{aligned}
$$

Q.E.D.Lemma 3

Proof of LEMMA 4. We begin with a claim.

Claim 19 Let $s$ be an increasing strategy profile satisfying

$$
\int_{z \in R^{I-1}} \pi_{i}(z) \Delta u_{i}\left(s_{i}\left(x_{i}\right), a_{i},\left(s_{j}\left(x_{i}+\nu z_{j}\right)\right)_{j \neq i}, x_{i}\right) d z \geq 0
$$

for all $i=1, \ldots, I, x_{i} \in \Re$ and $a_{i} \leq s_{i}\left(x_{i}\right)$.

Lemma 20 Then, for any $\varepsilon>0$ and for any compact interval $B$, there exists $\delta>0$, such that

$$
\int_{z \in R^{I-1}} \pi_{i}^{\prime}(z) \Delta u_{i}\left(s_{i}\left(x_{i}\right), a_{i},\left(s_{j}\left(x_{i}+\nu z_{j}\right)\right)_{j \neq i}, x_{i}+\varepsilon\right) d z>0,
$$

for all $i=1, \ldots, I, \pi_{i}^{\prime} \in \Psi_{\delta}\left(\pi_{i}\right), x_{i} \in B$ and $a_{i} \leq s_{i}\left(x_{i}\right)$.

Proof. For all $\delta>0$ and $\pi_{i}^{\prime} \in \Psi_{\delta}\left(\pi_{i}\right)$,

$$
\begin{aligned}
& \int_{z \in R^{I-1}} \pi_{i}^{\prime}(z) \Delta u_{i}\left(s_{i}\left(x_{i}\right), a_{i},\left(s_{j}\left(x_{i}+\nu z_{j}\right)\right)_{j \neq i}, x_{i}+\varepsilon\right) d z \\
& \geq\left\{\begin{array}{c}
(1-\delta) \int_{z \in R^{I-1}} \pi_{i}(z) \Delta u_{i}\left(s_{i}\left(x_{i}\right), a_{i},\left(s_{j}\left(x_{i}+\nu z_{j}\right)\right)_{j \neq i}, x_{i}+\varepsilon\right) d z \\
+\delta \Delta u_{i}\left(s_{i}\left(x_{i}\right), a_{i}, \mathbf{0}_{-i}, x_{i}+\varepsilon\right)
\end{array}\right\} \\
& \geq\left\{\begin{array}{l}
(1-\delta) \int_{z \in R^{I-1}} \pi_{i}(z)\left[\begin{array}{l}
\Delta u_{i}\left(s_{i}\left(x_{i}\right), a_{i},\left(s_{j}\left(x_{i}+\nu z_{j}\right)\right)_{j \neq i}, x_{i}+\varepsilon\right) \\
-\Delta u_{i}\left(s_{i}\left(x_{i}\right), a_{i},\left(s_{j}\left(x_{i}+\nu z_{j}\right)\right)_{j \neq i}, x_{i}\right)
\end{array}\right] d z \\
+(1-\delta) \int_{z \in R^{I-1}} \pi_{i}(z) \Delta u_{i}\left(s_{i}\left(x_{i}\right), a_{i},\left(s_{j}\left(x_{i}+\nu z_{j}\right)\right)_{j \neq i}, x_{i}\right) d z \\
+\delta \Delta u_{i}\left(s_{i}\left(x_{i}\right), a_{i}, \mathbf{0}_{-i}, \underline{\theta}\right)
\end{array}\right\}
\end{aligned}
$$


By A3, we can find a constant $K_{2}>0$ such that for all $x_{i}$ in the compact interval $B$, the first term is at least $(1-\delta) K_{2} \varepsilon\left(s_{i}\left(x_{i}\right)-a_{i}\right)$. The second term is at least 0 , by the premise of the lemma. By A6 there is a constant $K_{3}$ such that the third term is at least $-\delta K_{3}\left(s_{i}\left(x_{i}\right)-a_{i}\right)$. Thus the sum is at least $\left((1-\delta) K_{2} \varepsilon-\delta K_{3}\right)\left(s_{i}\left(x_{i}\right)-a_{i}\right)$. This expression must be positive (for all $\left.a_{i}<s_{i}\left(x_{i}\right)\right)$ if we choose $\delta$ such that $\frac{\delta}{1-\delta}<\frac{K_{2} \varepsilon}{K_{3}}$. Q.E.D.Claim 19

By construction of $\underline{s}$, we know that for all $b \geq \varepsilon, i=1, \ldots, I$ and $x_{i} \in \mathbf{R}$

$$
\int_{z \in R^{I-1}} \pi_{i}(z) \Delta u_{i}\left(\underline{s}_{i}\left(x_{i}-b\right), a_{i},\left(\underline{s}_{j}\left(x_{i}-b+\nu z_{j}\right)\right)_{j \neq i}, x_{i}-b\right) d z \geq 0
$$

for all $a_{i} \leq \underline{s}_{i}\left(x_{i}-b\right)$. This implies (by lemmas 3 and 19) that there exists $\widetilde{\nu}$ such that for all $\nu \leq \widetilde{\nu}, b \geq \varepsilon$ and $x_{i} \in \mathbf{R}$,

$$
\int_{z \in R^{I-1}} \tilde{\pi}_{i}\left(z \mid x_{i}, \nu\right) \Delta u_{i}\left(\underline{s}_{i}\left(x_{i}-b\right), a_{i},\left(\underline{s}_{j}\left(x_{i}-b+\nu z_{j}\right)\right)_{j \neq i}, x_{i}-\frac{b}{2}\right) d z \geq 0
$$

for all $a_{i} \leq \underline{s}_{i}\left(x_{i}-b\right){ }^{6}$ This implies (changing notation only) that

$$
\int_{\theta=-\infty}^{\infty} \int_{x_{-i} \in R^{I-1}} \widetilde{\phi}_{i}\left(x_{-i}, \theta \mid x_{i}\right) \Delta u_{i}\left(\underline{s}_{i}\left(x_{i}-b\right), a_{i},\left(\underline{s}_{j}\left(x_{j}-b\right)\right)_{j \neq i}, x_{i}-\frac{b}{2}\right) d x_{-i} d \theta \geq 0
$$

for all $a_{i} \leq \underline{s}_{i}\left(x_{i}-b\right)$, where

$$
\widetilde{\phi}_{i}\left(x_{-i}, \theta \mid x_{i}\right)=\frac{\phi(\theta) \prod_{j=1}^{I} f_{j}\left(\frac{x_{j}-\theta}{\nu}\right)}{\int_{\theta^{\prime}=-\infty}^{\infty} \phi\left(\theta^{\prime}\right) f_{i}\left(\frac{x_{i}-\theta^{\prime}}{\nu}\right) d \theta^{\prime}} .
$$

\footnotetext{
${ }^{6}$ Assuming A2, lemma 19 implies this for $x_{i}-b \in[\underline{\theta}, \bar{\theta}]$. For $x_{i}-b$ not in this interval, the result holds since $\underline{s}_{i}\left(x_{i}-b\right)$ must be either 0 or 1 , depending on which is dominant at the payoff parameter $x_{i}-b$. Assuming A2 $2^{\prime}$ instead of A2, lemma 19 implies this for $x_{i}-b \in\left[\underline{\theta}^{\prime}-\widetilde{\nu}, \bar{\theta}^{\prime}+\widetilde{\nu}\right]$, where $\underline{\theta}^{\prime}$ and $\bar{\theta}^{\prime}$ are defined near the end of the proof of proposition 2. Below $\underline{\theta}^{\prime}, \underline{a}$ must be played; above $\bar{\theta}^{\prime}, \bar{a}$ must be played. Thus, for $x_{i}-b<\underline{\theta}^{\prime}-\widetilde{\nu}, \underline{s}_{i}\left(x_{i}-b\right)=\underline{a}_{i}$ and $\underline{s}_{j}\left(x_{i}-b+\nu z_{j}\right)=\underline{a}_{j}$ for all $z_{j}$ that has positive probability under both $\pi_{i}(z)$ and $\tilde{\pi}_{i}\left(z \mid x_{i}, \nu\right)$. Hence, changing the distribution of $z$ leaves the integral unchanged. The same holds for $x_{i}-b>\bar{\theta}^{\prime}+\widetilde{\nu}$.
} 
Now if $\nu<\frac{b}{2}$ we have by assumption A3 that

$$
\int_{\theta=-\infty}^{\infty} \int_{x_{-i} \in R^{I-1}} \widetilde{\phi}_{i}\left(x_{-i}, \theta \mid x_{i}\right) \Delta u_{i}\left(\underline{s}_{i}\left(x_{i}-b\right), a_{i},\left(\underline{s}_{j}\left(x_{j}-b\right)\right)_{j \neq i}, \theta\right) d x_{-i} d \theta>0
$$

for all $a_{i}<\underline{s}_{i}\left(x_{i}-b\right)$. Set $\nu \leq \bar{\nu}=\min \left\{\frac{\varepsilon}{2}, \widetilde{\nu}\right\}$. Consider the strategy profile $s^{\prime}$ where $s_{i}^{\prime}\left(x_{i}\right)=\underline{s}_{i}\left(x_{i}-b\right)$. By equation 6 , we know that, in $G(\nu)$, each player's best response to $s^{\prime}$ is always at least $s^{\prime}$.

Since this is true for any $b \geq \varepsilon$, this ensures that iterated deletion of strictly dominated strategies (using translations of $\underline{s}$ as in the proof of lemma 2), cannot lead below $\underline{s}_{i}^{\nu}\left(x_{i}-\varepsilon\right)$. A symmetric argument (using a symmetric version of lemma 19, whose proof is analogous) gives the upper bound. Q.E.D.Lemma 4 


\section{References}

[1] Burdzy, Krzysztof, David M. Frankel, and Ady Pauzner. 1999. "Fast Equilibrium Selection by Rational Players Living in a Changing World." Forthcoming, Econometrica. Available at www.tau.ac.il/ pauzner

[2] Carlsson, H. (1989). "Global Games and the Risk Dominance Criterion."

[3] Carlsson, H. and E. van Damme (1993a). "Global Games and Equilibrium Selection," Econometrica 61, 989-1018.

[4] Carlsson, H. and E. van Damme (1993b). "Equilibrium Selection in Stag Hunt Games," in Frontiers of Game Theory (K. Binmore, A. Kirman and A. Tani, Eds.). Cambridge, MA: MIT Press.

[5] Carlsson, H. and M. Ganslandt (1998). "Noisy Equilibrium Selection in Coordination Games," Economics Letters 60, 23-34.

[6] Frankel, David M. 2000. "Dynamic Equilibrium Selection: A General Uniqueness Result." Available at www.tau.ac.il/ ^dfrankel/papers.html

[7] Frankel, David M., and Ady Pauzner. 1999a. "Resolving Indeterminacy in Dynamic Settings: the Role of Shocks." Forthcoming, Quarterly Journal of Economics. Available at www.tau.ac.il/ ${ }^{\sim}$ pauzner

[8] Frankel, David M., and Ady Pauzner. 1999b. "Expectations and the Timing of Neighborhood Change." Mimeo. Available at www.tall.ac.il/ dfrankel/papers.html

[9] Harsanyi, J. and R. Selten (1988). A General Theory of Equilibrium Selection in Games. Cambridge: M.I.T. Press.

[10] Heinemann, F. (1999). "Unique Equilibrium in a Model of Self-Fulfilling Currency Attacks: Comment," forthcoming in American Economic Review. 
[11] Kajii, A. and S. Morris (1997). "The Robustness of Equilibria to Incomplete Information," Econometrica 65, 1283-1309.

[12] Kim, Y. (1996). "Equilibrium Selection in N-Person Coordination Games," Games and Economic Behavior 15, 203-227.

[13] Monderer, D. and L. Shapley (1996). "Potential Games," Games and Economic Behavior 14, 124-143.

[14] Morris (1999). "Potential Methods in Interaction Games."

[15] Morris, S., R. Rob and H. S. Shin (1995). " $p$-Dominance and Belief Potential," Econometrica $63,145-157$.

[16] Morris, S. and H. S. Shin (1998). "Unique Equilibrium in a Model of Self-Fulfilling Currency Attacks," American Economic Review 88, 587-597.

[17] Morris, S. and H. S. Shin (1999). "Coordination Risk and the Price of Debt." 